\title{
Monte Carlo approximations of the Neumann problem
}

\author{
Sylvain Maire* and Etienne Tanré ${ }^{\dagger}$
}

August 21, 2013

\begin{abstract}
We introduce Monte Carlo methods to compute the solution of elliptic equations with pure Neumann boundary conditions. We first prove that the solution obtained by the stochastic representation has a zero mean value with respect to the invariant measure of the stochastic process associated to the equation. Pointwise approximations are computed by means of standard and new simulation schemes especially devised for local time approximation on the boundary of the domain. Global approximations are computed thanks to a stochastic spectral formulation taking into account the property of zero mean value of the solution. This stochastic formulation is asymptotically perfect in terms of conditioning. Numerical examples are given on the Laplace operator on a square domain with both pure Neumann and mixed Dirichlet-Neumann boundary conditions. A more general convection-diffusion equation is also numerically studied.
\end{abstract}

Keywords. Neumann Problem, Monte Carlo Methods, Spectral Methods, Local Time Approximation

\section{Introduction}

The aim of this paper is to compute pointwise and global numerical approximations of the solutions of pure Neumann problems for elliptic equations by means of Monte Carlo procedures. The pointwise solutions will be obtained via Feynman-Kac representations and the global ones by stochastic spectral formulations inspired from the methods developed in our previous works [18, 19].

We first consider the Neumann problem for the Laplace operator in a domain $D \subset \mathbb{R}^{d}$ which writes

$$
\left\{\begin{array}{l}
\frac{1}{2} \Delta u(x)=-f(x) \quad \text { for } \quad x=\left(x_{1}, \ldots, x_{d}\right) \in D \\
\frac{1}{2} \frac{\partial u}{\partial n}(y)=g(y) \text { for } y \in \partial D,
\end{array}\right.
$$

${ }^{*}$ Laboratoire des Sciences de l'Information et des Systemes (LSIS), UMR6168, ISITV, Université de Toulon et du Var, Avenue G. Pompidou, BP 56, 83262 La Valette du Var cedex, France sylvain.maire@univ-tln.fr

${ }^{\dagger}$ INRIA, EPI Tosca, 2004 route des Lucioles BP93 F-06902 Sophia-Antipolis - France Etienne.Tanre@inria.fr 
where $\frac{\partial u}{\partial n}$ stands for the incoming normal derivative of $u$ on the boundary of the domain $D$. Because the boundary conditions are of Neumann type everywhere on the boundary, this equation has a solution up to an additive constant whenever an additional compatibility condition on $f$ and $g$ is satisfied (see [10]). This compatibility condition writes

$$
\int_{D} f(x) d x+\int_{\partial D} g(y) d y=0 .
$$

In the case of the Poisson equation with Dirichlet boundary conditions, the probabilistic representations of the solutions are based on Feynman-Kac formulae which are stopped at the first hitting time of the boundary by a Brownian motion. In the case of mixed boundary conditions, that is of Neumann type on a part of the boundary and of Dirichlet type on the other part, the (reflected) Brownian motion is still stopped at the first hitting time of the Dirichlet boundary. In the case of pure Neumann boundary conditions the Brownian motion never stops and the probabilistic representation introduced by Brosamler [6] in the case $f=0$ is the limit in time of the Feynman-Kac representation of the solution of the relative Cauchy problem. We have

$$
u(x)=\lim _{T \rightarrow \infty} \mathbb{E}_{x}\left(\int_{0}^{T} g\left(B_{s}\right) d V_{s}\right)
$$

where $\left(B_{t}, t \geq 0\right)$ stands for the reflected Brownian motion in $D$ and $\left(V_{t}, t \geq 0\right)$ is its associated local time on the boundary. The proof of this representation is based on probabilistic potential theory combined with a representation of additive functionals. This representation has been extended by Benchérif-Madani and Pardoux [2] to more general advection-diffusion equations

$$
L u(x)=-f(x),
$$

with boundary conditions $\frac{\partial u}{\partial n_{a}}(y)=g(y)$, where

$$
L u(x)=\frac{1}{2} \sum_{i, j=1}^{d} a_{i, j}(x) \frac{\partial^{2} u(x)}{\partial x_{i} \partial x_{j}}+\sum_{i=1}^{d} b_{i}(x) \frac{\partial u(x)}{\partial x_{i}}
$$

and $\frac{\partial u}{\partial n_{a}}()=.\frac{1}{2} a \nabla(.) \cdot n$ stands for the conormal derivative. Some regularity assumptions are required on the domain $D$, the functions $f$ and $g$, the coefficients $a_{i, j}$ and $b_{i}$ and also uniform ellipticity conditions and boundness for the symmetric matrix $a$. We have

$$
u(x)=\lim _{T \rightarrow \infty} u(x, T)=\lim _{T \rightarrow \infty} \mathbb{E}_{x}\left(\int_{0}^{T} f\left(X_{s}\right) d s+\int_{0}^{T} g\left(X_{s}\right) d V_{s}\right)
$$

where $\left(X_{t}, t \geq 0\right)$ is the reflected diffusion process and $u(x, T)$ the solution of the Cauchy problem associated to the operator $L$. The compatibility condition becomes

$$
\int_{D} f(x) p(x) d x+\int_{\partial D} g(y) p(y) d y=0
$$


where $p(x)$ is the density of the invariant measure associated to $\left(X_{t}, t \geq 0\right)$. The proof of this more general representation is now based on exponential ergodicity.

We intend to use the previous stochastic representation for the numerical computation of the Neumann problem by means of Monte Carlo procedures. We need to overcome several problems. To compute the pointwise solution, we have to choose a time $T_{0}$ to stop the trajectories, deal efficiently with the approximation of the Neumann boundary conditions (which involves local time approximation) and understand which additive constant is obtained using this representation. The Monte Carlo approximation of the Neumann problem associated to the Laplace operator has already been treated using the walk on the boundary method [25]. The truncation problems of the divergent Neumann serie linked to this walk are very similar to the choice of the stopping time $T_{0}$ of our trajectories. This method deals very naturally with the Neumann boundary conditions but may suffer of an increase of the variance for the computation of the solution at points close to the boundary of the domain. Some estimators with a reduced variance are also proposed in 25. They require to find the part of the boundary where the boundary term is positive. To obtain the global solution, we need to choose and to build carefully the basis functions involved in the stochastic spectral formulations in order to obtain an unique and well characterized solution. The rest of the paper is organized as follows.

In section 2, we first prove that the representation of Bencherif-Madani and Pardoux [2] naturally leads to a solution $u(x)$ such that its mean value with respect to the invariant measure is zero. For a practical computation, we need to replace the limit in representation (1) by a finite time $T_{0}$. This introduces a bias that will be linked to the second eigenvalue of the operator $L$ with pure Neumann boundary conditions. The question of the variance of our estimators as a function of $T_{0}$ is also studied. We remark that the variance has a linear growth as a function of $T_{0}$.

In section 3, we describe a general algorithm to approximate the solution of general elliptic Neumann problems by means of the Euler scheme coupled with a local time approximation method developed in [16].

Section 4 is devoted to walk on spheres algorithms which can be used in the special case of the Poisson equation. We describe how to adapt them to a Cauchy problem with a finite horizon $T_{0}$. Furthermore, two new schemes with an increased order of convergence are introduced to deal with inhomogeneous Neumann boundary conditions.

In section 5, we give some numerical results on mixed Dirichlet-Neumann problems and on pure Neumann problems with different degrees of difficulty. For the pure Neumann problem, the scheme that is used to compute the representation modifies the additive constant from which the solution depends.

Finally in section 6, we develop stochastic spectral methods in order to obtain a very accurate and global approximation of the solution. In addition to the general methodology developed in [18, 19], we need to develop centering procedures for our approximation basis in order to project our solution in the space with zero mean value with respect to $p(x)$. Numerical examples show that our method is very efficient in terms of accuracy and conditioning in the both 
cases where the invariant measure is known analytically or only approximated.

\section{Some properties of the stochastic representa- tion}

For theoretical purpose, representation (1) is useful. For a numerical purpose, we need to understand which solution is effectively computed (not only up to an additive constant) and the consequences of replacing the limit by a fixed time $T_{0}$. We shall prove in the following that the solution $u(x, T)$ has asymptotically a mean value zero with respect to the invariant measure and that its variance is increasing mainly linearly as a function of $T$.

We consider a general diffusion operator $L$ verifying the properties mentioned in the introduction [2]. We denote by $\left(X_{t}, t \geq 0\right)$ its associated reflected diffusion process

$$
X_{t}=x+\int_{0}^{t} b\left(X_{s}\right) d s+\int_{0}^{t} \sigma\left(X_{s}\right) d W_{s}-\frac{1}{2} \int_{0}^{t} \gamma\left(X_{s}\right) d V_{s}
$$

where the matrix $\sigma$ is such that $\sigma \sigma^{T}=a,\left(W_{t}, t \geq 0\right)$ is a $d$ dimensional Brownian motion, $\left(V_{t}, t \geq 0\right)$ is the local time on the boundary and $\gamma$ is in the conormal direction

$$
\gamma(x)=a n(x) .
$$

We then define the solution $u(x, T)$ of the Cauchy problem

$$
u(x, T)=\mathbb{E}_{x}\left(\int_{0}^{T} f\left(X_{s}\right) d s+\int_{0}^{T} g\left(X_{s}\right) d V_{s}\right)
$$

where $f$ and $g$ are bounded and verify the compatibility condition

$$
\int_{D} f(x) p(x) d x+\int_{\partial D} g(y) p(y) d y=0 .
$$

Following the arguments developed in [2], there exists a bounded function $f_{1}$ such that

$$
u(x, T)=\mathbb{E}_{x}\left(\int_{0}^{T} f_{1}\left(X_{s}\right) d s\right)
$$

with the new compatibility condition

$$
\int_{D} f_{1}(x) p(x) d x=0
$$

\subsection{Characterization of the solution}

We can first notice that

$$
\int_{D} \mathbb{E}_{x}\left(f_{1}\left(X_{s}\right)\right) p(x) d x=\mathbb{E}_{\mu}\left(f_{1}\left(X_{s}\right)\right)=\int_{D} f_{1}(x) p(x) d x=0
$$


for any time $s$ because $\mu$ is the invariant law for (2). Using the Fubini theorem, this leads to

$$
\int_{D} u(x, T) p(x) d x=0
$$

for any time $T$. In the case of the Laplace operator, it has been proven in [1] that the convergence in time of $u(x, T)$ towards $u(x)$ is uniform in $x$ and thus the desired property holds. This uniform convergence relies on special properties of the Brownian motion that cannot be extended so easily to a general diffusion process. These properties are a bound on the transition density function $p(t, x, y)$ of the reflecting Brownian motion and its spectral expansion [7]. This spectral expansion writes

$$
p(t, x, y)=\frac{1}{|D|}+\sum_{n=1}^{\infty} \exp \left(-\lambda_{n} t\right) \phi_{n}(x) \phi_{n}(y)
$$

where $\frac{1}{|D|}$ is the uniform measure in $D,\left(\lambda_{n}, n \geq 0\right)$ and $\left(\phi_{n}, n \geq 0\right)$ the eigenelements of $-\frac{1}{2} \Delta$. The first term $\frac{1}{|D|}$ corresponds to the first eigenvalue $\lambda_{0}=0$ and

$$
\phi_{0}(x)=\frac{1}{\sqrt{D}} .
$$

The remaining eigenvalues $\left(\lambda_{n}, n \geq 1\right)$ are strictly positive. Theorem 2.4 of [1] says that $p(t, x, y)$ converges exponentially fast and uniformly to $\frac{1}{|D|}$ at a speed linked to the second eigenvalue $-\lambda_{1}$ of $\frac{1}{2} \Delta$. This can guide us for the choice of the time $T_{0}$ at which we should stop our trajectories.

In the general case, we have for any $T \geq 0$,

$$
\left|\int_{D} u(x) p(x) d x\right|^{2}=\left|\int_{D} u(x) p(x) d x-\int_{D} u(x, T) p(x) d x\right|^{2} \leq \int_{D}(u(x)-u(x, T))^{2} p(x) d x
$$

thanks to the Cauchy-Schwarz inequality. In lemma 3 of [2], it is proven an exponential ergodicity for the solution $w(x, t)=\mathbb{E}_{x}\left(f_{1}\left(X_{t}\right)\right)$ of the parabolic problem with an initial condition $f_{1}$ satisfying the centering condition, no source term and homogeneous Neumann boundary conditions. This is summarized in the inequality

$$
\int_{D} w(x, t)^{2} p(x) d x \leq \exp (-r t) \int_{D} f_{1}(x)^{2} p(x) d x
$$

where $r$ is a positive constant. We can write

$$
\int_{D}(u(x)-u(x, T))^{2} p(x) d x \leq \int_{D} p(x) d x \int_{T}^{\infty} \exp \left(\frac{r}{2} s\right) w(x, s)^{2} d s \times \int_{T}^{\infty} \exp \left(-\frac{r}{2} s\right) d s
$$

thanks again to the Cauchy-Schwarz inequality and finally

$$
\left|\int_{D} u(x) p(x) d x\right| \leq \frac{2}{r} \exp (-r T) \int_{D} f_{1}(x)^{2} p(x) d x
$$

thanks to the Fubini theorem and to the exponential ergodicity bound. This proves that $\int_{D} u(x) p(x) d x=0$. 


\subsection{Estimation of the variance}

In this section, we estimate the variance of $\int_{0}^{T} f_{1}\left(\bar{B}_{s}\right) d s$ as a function of $T$ in the simplified case of the Poisson equation in dimension one in the interval $D=[a, b]$ with boundary conditions $u^{\prime}(a)=u^{\prime}(b)=0$. We have

$$
\operatorname{Var}\left(\int_{0}^{T} f_{1}\left(\bar{B}_{s}\right) d s\right)=\mathbb{E}_{x}\left(\int_{0}^{T} f_{1}\left(\bar{B}_{s}\right) d s\right)^{2}-\left(\mathbb{E}_{x} \int_{0}^{T} f_{1}\left(\bar{B}_{s}\right) d s\right)^{2}
$$

where $\left(\bar{B}_{s}, s \geq 0\right)$ is a reflected Brownian motion in $D$. As

$$
\lim _{T \rightarrow \infty}\left(\mathbb{E}_{x} \int_{0}^{T} f_{1}\left(\bar{B}_{s}\right) d s\right)^{2}=u^{2}(x),
$$

the second term in the variance is obviously bounded because $u$ is a continuous function in a bounded domain. For the first term, thanks to Itô-Tanaka formula we have

$u\left(\bar{B}_{T}\right)=u(x)+\int_{0}^{T} L u\left(\bar{B}_{s}\right) d s+\int_{0}^{T} u^{\prime}\left(\bar{B}_{s}\right) d B_{s}=u(x)-\int_{0}^{T} f_{1}\left(\bar{B}_{s}\right) d s+\int_{0}^{T} u^{\prime}\left(\bar{B}_{s}\right) d B_{s}$.

Thanks to the boundary conditions, the usual term involving the local time process is equal to zero. The above formula leads to

$$
\begin{aligned}
\left(\int_{0}^{T} f_{1}\left(\bar{B}_{s}\right) d s\right)^{2}= & \left(u(x)-u\left(\bar{B}_{T}\right)\right)^{2}+\left(\int_{0}^{T} u^{\prime}\left(\bar{B}_{s}\right) d B_{s}\right)^{2} \\
& +2\left(u(x)-u\left(\bar{B}_{T}\right)\right)\left(\int_{0}^{T} u^{\prime}\left(\bar{B}_{s}\right) d B_{s}\right) .
\end{aligned}
$$

Using that the invariant measure is uniform in $D$, we have

$$
\lim _{T \rightarrow \infty} \mathbb{E}_{x}\left[\left(u(x)-u\left(\bar{B}_{T}\right)\right)\right]^{2}=\frac{1}{|D|} \int_{D}(u(x)-u(y))^{2} d y
$$

which proves that this term is bounded and its limit identified. Then from the isometry property

$$
\mathbb{E}_{x}\left[\left(\int_{0}^{T} u^{\prime}\left(\bar{B}_{s}\right) d B_{s}\right)^{2}\right]=\mathbb{E}_{x}\left[\int_{0}^{T}\left(u^{\prime}\left(\bar{B}_{s}\right)\right)^{2} d s\right]
$$

and from the ergodic theorem

$\mathbb{E}_{x}\left[\left(\int_{0}^{T} u^{\prime}\left(\bar{B}_{s}\right) d B_{s}\right)^{2}\right]=T \mathbb{E}_{x}\left[\frac{1}{T} \int_{0}^{T}\left(u^{\prime}\left(\bar{B}_{s}\right)\right)^{2} d s\right] \simeq T \frac{1}{|D|} \int_{D}\left(u^{\prime}(y)\right)^{2} d y$.

Thanks now to the Cauchy-Schwarz inequality and to the previous bounds 


$$
\left|\mathbb{E}_{x}\left(u(x)-u\left(\bar{B}_{T}\right)\right)\left(\int_{0}^{T} u^{\prime}\left(\bar{B}_{s}\right) d B_{s}\right)\right| \leq C \sqrt{T}\left(\frac{1}{|D|} \int_{D}\left(u^{\prime}(y)\right)^{2} d y\right)^{1 / 2}
$$

which proves finally that asymptotically

$$
C_{1}-C_{2} \sqrt{T}+C_{3} T \leq \operatorname{Var}\left(\int_{0}^{T} f\left(\bar{B}_{s}\right) d s\right) \leq C_{1}+C_{2} \sqrt{T}+C_{3} T
$$

for some constants $C_{1}, C_{2}$ and where $C_{3}=\frac{1}{|D|} \int_{D}\left(u^{\prime}(y)\right)^{2} d y$. The lower and upper bounds on the variance show that it increases essentially as a linear function of $T$ (except if $u(x)$ is constant). This proves that the time chosen to stop the trajectories is both crucial in terms of bias and in terms of variance.

\section{$3 \quad$ Euler scheme approximations}

We now present a scheme used to approximate the solution $u$ inspired by the representation (1) but where the limit is replaced by a fixed finite time $T_{0}$. This method relies on weak approximations. First of all, in a general setting, we have to approximate the reflected diffusion process $\left(X_{t}, 0 \leq t \leq T_{0}\right)$ associated to the infinitesimal generator $L$ in the domain $D \subset \mathbb{R}^{d}$. The approximation of diffusion processes is usually done using the Euler scheme. The errors and the error expansions on the weak approximations using this scheme in the whole space have been first studied in [27. The weak approximation of diffusions with absorbing (Dirichlet) and reflected (Neumann) boundary conditions have been treated respectively in [11] and [5]. Given a time step $\delta$, the approximation of $\left(X_{t}, t \geq 0\right)$ by a standard reflected Euler scheme $\left(\bar{X}_{k \delta}, 0 \leq k \leq T_{0} / \delta\right)$ can be described by the following procedure.

1. for all $i=1 . . d$,

$$
\tilde{X}_{(k+1) \delta}^{i}=\bar{X}_{k \delta}^{i}+b_{i}\left(\bar{X}_{k \delta}\right) \delta+\sum_{j} \sigma_{i, j}\left(\bar{X}_{k \delta}\right)\left(W_{(k+1) \delta}^{j}-W_{k \delta}^{j}\right) .
$$

2. if $\tilde{X}_{(k+1) \delta} \in D$, we set $\bar{X}_{(k+1) \delta}=\tilde{X}_{(k+1) \delta}$,

3. else, if $\tilde{X}_{(k+1) \delta} \notin D$, we have to choose another position inside the domain $D$. The final position $\bar{X}_{(k+1) \delta}$ is the symmetrized of the Euler scheme $\tilde{X}_{(k+1) \delta}$ in the conormal direction a.n (see [5]).

To compute our representation, we first need to approximate the integral $\int_{0}^{T_{0}} f\left(X_{s}\right) d s$. This can be done using the rectangle method by the discrete sum

$$
\delta \sum_{k=0}^{\frac{T_{0}}{\delta}-1} f\left(\bar{X}_{k \delta}\right)
$$


The approximation of the term $\int_{0}^{T_{0}} g\left(X_{s}\right) d V_{s}$ is less classical (see e.g. [16]). We introduce a delocalization parameter $\xi$ and compute the approximation

$$
\delta \sum_{k=0}^{\frac{T_{0}}{\delta}-1} g\left(\pi\left(\bar{X}_{k \delta}\right)\right) K_{\xi}\left(\bar{X}_{k \delta}, \pi\left(\bar{X}_{k \delta}\right)\right)
$$

where $K_{\xi}$ stands for instance for the Gaussian kernel

$$
K_{\xi}(x, y)=\frac{1}{(2 \pi)^{d / 2} \xi^{d}} \exp \left(-\sum_{i=1}^{d} \frac{\left(x_{i}-y_{i}\right)^{2}}{\xi^{2}}\right)
$$

We have also used the orthogonal projection $\pi$ on the boundary $\partial D$. The delocalization parameter must be chosen carefully. We will observe in section 5 that it should not be too small unless the variance becomes very large. In this section, we will also consider a problem with mixed boundary conditions. The treatment of the Dirichlet boundary conditions will rely on half-space approximations [12. An additional stopping test of the trajectories based on a Brownian bridge enables to obtain an order 1 weak error in $\delta$ on the part of the boundary with Dirichlet boundary conditions. The layer method described in 22 can also be used. It is especially adapted to parabolic problems and enables to obtain very accurate approximations of such problems. It relies however on a Markov chain which is more difficult to simulate than the Euler scheme.

\section{Walk on spheres approximations}

\subsection{Introduction}

The Euler scheme can be used for the simulation of a wide class of stochastic processes linked to second order elliptic operators. If we deal with the Laplace operator or with divergence form operators with constant or piecewise constant diffusion coefficients [17, more efficient simulations of Brownian paths are available like walk on spheres (WOS) [24] or walk on rectangles algorithms 9 .

In the case of Dirichlet boundary conditions, the stochastic representation of the solution implies a Brownian path up to the first time it hits the boundary $\partial D$ of the domain. To solve the Neumann problem, we should use the same scheme as soon as the particle is away from the boundary, that is it has not reached the $\varepsilon$-absorption layer. Then, we replace the particle inside the domain and run again the same scheme until hitting one more time the boundary and so on. Some efficient ways to replace the particle inside the domain are described in section 4.3

Let us now describe the WOS method to compute the solution of the Laplace equation at a point $x \in D$. The walk starts at $x$ and jumps from one sphere to another until it reaches an $\varepsilon$-absorption layer $(=\{y \in D, d(y, \partial D) \leq \varepsilon\})$. The spheres are built so that the jumps are as large as possible by taking the radius of the next sphere as the distance to the boundary $\partial D$. The next point is the 
first hitting point of the sphere by a Brownian motion started at the center. So, thanks to the isotropy of the Brownian motion, it has to be chosen uniformly on this sphere. We stop the walk at the first time the selected point on the sphere belongs to the $\varepsilon$-absorption layer. The contribution of each walk to the solution is the value of the boundary term at a projection (generally the orthogonal one) of the current position on the boundary. Finally, the approximate solution is the average of the contributions of walks. In the case of the Poisson equation, it is also possible to compute the contribution of the source term during this walk [15] using for each sphere $S$ of radius $r_{n}$ and center $x_{n}$ a Green function $G$ conditioned by the exit point $z$ writing

$$
\mathbb{E}_{x_{n}}\left(\int_{0}^{\tau_{S}} f\left(B_{t}\right) d t \mid B_{\tau_{S}}=z\right)=\frac{r_{n}^{2}}{d} \int_{S} G(z, y) f(y) d y .
$$

At least in dimension 2, the conditional density is known exactly and it is possible to sample easily from it. Thus we can obtain the contribution of the source term to the whole trajectory.

\subsection{WOS for the pure Neumann problem}

Regardless the problem of making a good choice of a final time $T_{0}$ to stop our trajectories, our task is to deal efficiently with the inhomogeneous Neumann boundary conditions while simulating accurately the elapsed time and position from the start of the trajectories. The description of the tools used when the walk hits the boundary is given in the next subsection. We need to adapt the WOS method to the case of a finite horizon $T_{0}$. First, formula (3) gives not

the exit time $\tau_{S}$ but only the average time $\frac{r_{n}^{2}}{d}$ spent in the sphere $S$ of radius $r_{n}$. Second, we want to approximate $\int_{0}^{\tau_{S}} f\left(B_{t}\right) d t$ knowing that $B_{\tau_{S}}=z$. It is possible to sample from $\tau_{S}$ using for instance the inverse method as its law has a closed form given by a serie. However, we need here to compute more complicated quantities involving positions of the Brownian motion before it hits the boundary conditioned by the value of $\tau_{S}$. We actually do not know closed forms for these quantities so we sample from them using precomputed discretized trajectories as described as follows. Note that anyway sampling from a law that is given by a serie by the inverse method requires an iterative method which may be time consuming compared to precomputation. The previous integral can be transformed writing

$$
\int_{0}^{\tau_{S}} f\left(B_{t}\right) d t=\tau_{S} \mathbb{E}^{U}\left[f\left(B_{U \tau_{S}}\right)\right]
$$

where $U$ is an uniform r.v. on $[0,1]$ independent of $\left(B_{t}, 0 \leq t \leq \tau_{S}\right)$ and $\mathbb{E}^{U}$ stands for the expectation with respect to $U$. This transformation called the one random point method has been used in our papers [18, 19] and also in [23] in the context of financial mathematics. Its interest is to give a lot cheaper evaluation of the integral for only a small increase of the variance. 
Thanks to the isotropy of the Brownian Motion, we only need to simulate the couple $\left(\tau_{S_{1}}, B_{U \tau_{S_{1}}}\right)$ knowing that $B_{\tau_{S_{1}}}=(1,0)$ in the unit circle. To do this, we simulate an absorbed Brownian motion starting at the center of the unit circle using the Euler scheme with a very small time step $\delta$ and the half space approximation [12]. The walk stops after a random number $M$ of steps at an exit point $z=\exp (i \theta)$. The exit time $\tau_{S}$ is approximated by $M \delta$, a point $(x, y)$ is picked uniformly at random among the $M$ points of the discretized trajectory and is rotated using a rotation of angle $-\theta$. We can thus obtain the empirical joint law of $\left(\tau_{S_{1}}, B_{U \tau_{S_{1}}}\right)$ knowing that $B_{\tau_{S_{1}}}=(1,0)$. Samples from this empirical law are precomputed and stored in a large list. To sample from the couple $\left(\tau_{S_{1}}, B_{U \tau_{S_{1}}}\right)$, we pick uniformly at random a couple (exit time, position) in the list and an angle $\alpha$ uniformly in $[0,2 \pi]$ to rotate the position of the previous couple.

Dealing with the pure Neumann problem, we have to take care to another difference with the resolution of the Dirichlet problem: at the last step, the final time $T_{0}$ is before the exit time of the sphere. Assuming that time is reinitialized to zero before the last sphere, we have to compute integrals of the form $\int_{0}^{T_{1}} f\left(B_{s}\right) d s$ knowing that $B_{\tau_{S}}=z$ and $T_{1} \leq \tau_{S}$. So, we also need to store $Q$ full trajectories (time, position) before the exit time $\tau_{S}$ to approximate the above integrals.

\subsection{Replacement after hitting the boundary}

It remains to deal with the inhomogeneous Neumann boundary conditions. When the process reaches the $\varepsilon$-absorption layer it is projected on the boundary $\partial D$ at a point $(x, y)$. The standard way to replace the process inside the domain is based on a finite differences approximations [20. The idea is to use a normal approximation of the derivative at the boundary. In order to simplify the description, we assume for instance that the boundary is locally vertical that is it has the form $\left((x, z), y_{\min } \leq z \leq y_{\max }\right)$. Let $(x, y)$ be a point on this part of boundary, we have the order one approximation

$$
\frac{1}{2} \frac{u(x, y)-u(x+h, y)}{h} \simeq g(x, y)
$$

which leads to $u(x, y) \simeq 2 h g(x, y)+u(x+h, y)$. This simply means in terms of randomization that $2 h g(x, y)$ is added to the score of the walk and that the process starts again in the domain at position $(x+h, y)$. This approach does not give the elapsed time from position $(x, y)$ to position $(x+h, y)$ and neither takes into account that we deal with the Laplace operator. Another approach based on the randomization of an integral equation has been introduced in [26]. This approach has an improved rate of convergence and can also deal with transmission conditions. We propose now two alternative methods one based on a kinetic approximation and the other on higher order finite differences. 


\subsubsection{Kinetic approximation}

A new scheme based on a kinetic approximation at the boundary has been introduced in [17] for the simulation of multidimensional diffusions in a media where the diffusion coefficient present some discontinuities. The homogeneous Neumann boundary conditions were also treated using this new scheme on a hard test case taken from the couplex exercices. This scheme is based on a small parameter approximation of the diffusion operator by a neutron transport operator. Another application was the Monte Carlo solution of the PoissonBoltzmann equation in molecular dynamics [4. All the numerical tests were very satisfactory compared to the finite differences approach. The new scheme is proven to have an increased order of convergence on the test case of a single sphere for the Poisson-Boltzmann equation.

We shall now describe this kinetic approximation in dimension 2 and how to adapt it to the treatment of inhomogeneous Neumann boundary conditions. We assume for the sake of simplicity that the process has hitten from the right a vertical boundary with a Neumann condition at a point $(x, y)$ which is infinitely away from the other boundaries. We introduce a small parameter $h$ in order to approximate the Laplace operator by the transport operator. We also pick a collision time $t_{c}$ according to an exponential law of parameter 1 and a velocity $\left(v_{x}=\cos \theta, v_{y}=\sin \theta\right)$ uniformly on the half unit circle $\left(-\frac{\pi}{2} \leq \theta \leq \frac{\pi}{2}\right)$. The new position is $\left(x+h v_{x} t_{c}, y+h v_{y} t_{c}\right)$ and the elapsed time is $h^{2} t_{c}$. To deal with Neumann boundary conditions, we can write the Taylor expansion

$$
u\left(x+h v_{x} t_{c}, y+h v_{y} t_{c}\right)=u(x, y)+h \psi_{1}(x, y)+h^{2} \psi_{2}(x, y)+O\left(h^{3}\right)
$$

with

$$
\psi_{1}(x, y)=-2 v_{x} t_{c} g(x, y)+v_{y} t_{c} \frac{\partial u(x, y)}{\partial y}
$$

and

$$
\psi_{2}(x, y)=\frac{1}{2}\left(v_{x}^{2} t_{c}^{2} \frac{\partial^{2} u(x, y)}{\partial x^{2}}+v_{y}^{2} t_{c}^{2} \frac{\partial^{2} u(x, y)}{\partial y^{2}}+2 v_{x} v_{y} t_{c}^{2} \frac{\partial^{2} u(x, y)}{\partial x \partial y}\right) .
$$

Taking its mean value, we obtain

$$
u(x, y)=\mathbb{E}\left(u\left(x+h v_{x} t_{c}, y+h v_{y} t_{c}\right)\right)+\frac{4 g(x, y)}{\pi} h+f(x, y) h^{2}+O\left(h^{3}\right)
$$

because

$$
\mathbb{E}\left(v_{y}\right)=0, \quad \mathbb{E}\left(v_{x}\right)=\int_{-\frac{\pi}{2}}^{\frac{\pi}{2}} \cos \theta d \theta, \quad \mathbb{E}\left(t_{c}^{2}\right)=2 .
$$

Our scheme is the randomization of the previous formula: the motion continues at point $\left(x+h v_{x} t_{c}, y+h v_{y} t_{c}\right)$ and the quantity

$$
\frac{4 g(x, y)}{\pi} h+f(x, y) h^{2}
$$


is added to the current score of the walk and $h^{2}$ to the total time. The error is a $O\left(h^{3}\right)$ each time the boundary is hitten during the walk if we do not take into account the other boundaries. If $(x, y)$ is close to another part of the boundary, the point

$$
\left(x+h v_{x} t_{c}, y+h v_{y} t_{c}\right)
$$

may be outside the domain. In this case, we reduce $h$ iteratively by a factor 2 until it is inside. In the case of a general boundary, the choice of the velocity law must be obviously adapted to the form of the boundary to ensure that the process reenters in $D$.

\subsubsection{Order three finite differences approximation}

Some more sophisticated finite differences schemes can also be considered. Given a step $h$, we approximate the Laplace operator and the normal derivative at the point $(x, y)$ of the boundary using order 3 finite differences. For the Laplace operator, we have thanks to the so-called order 2 diamond scheme

$-\frac{1}{2} \Delta u(x, y)=f(x, y) \simeq \frac{-u(x-h, y)-u(x, y-h)-u(x, y+h)-u(x+h, y)+4 u(x, y)}{2 h^{2}}$

and for the centered normal derivative

$$
\frac{1}{2} \frac{\partial u(x, y)}{\partial x} \simeq \frac{u(x+h, y)-u(x-h, y)}{4 h}=g(x, y)+O\left(h^{2}\right) .
$$

By combining these two equations and getting rid of the fictitious value $u(x-$ $h, y)$, we obtain

$u(x, y)=\frac{2 u(x+h, y)+u(x, y+h)+u(x, y-h)}{4}+\frac{h^{2}}{2} f(x, y)+2 h g(x, y)+O\left(h^{3}\right)$.

This formula can be used for Monte Carlo simulations. Each time the Brownian motion hits the boundary at a point $(x, y)$, the quantity

$$
\frac{h^{2}}{2} f(x, y)+2 h g(x, y)
$$

is added to the current score and the motion continues at one of the positions

$$
(x+h, y),(x, y+h),(x, y-h)
$$

with the discrete probability law $\left(\frac{1}{2}, \frac{1}{4}, \frac{1}{4}\right)$. Some algorithms of a similar type for a walk on a grid have been introduced in 21] and are called sliding on the boundary methods because some of the possible points of replacement are on the boundary.

With our approach, if the boundary is not a straight line, the points $(x, y+h)$ and $(x, y-h)$ may lie outside the domain. If it happens, one needs to project the point on the boundary which induces an additional error. We introduce 
now a new similar scheme for a general boundary which avoids this projection. Instead of writing the previous equations at point $(x, y)$, we write them at point $(x+h, y)$. We obtain

$$
\frac{-u(x, y)-u(x+h, y-h)-u(x+h, y+h)-u(x+2 h, y)+4 u(x+h, y)}{2 h^{2}} \simeq f(x+h, y)
$$

for the Laplace operator. For the normal derivatives, we write the two Taylor expansions

$$
u(x+h, y)=u(x, y)+h \frac{\partial u(x, y)}{\partial x}+\frac{h^{2}}{2} \frac{\partial^{2} u(x, y)}{\partial x^{2}}+O\left(h^{3}\right)
$$

and

$$
u(x+2 h, y)=u(x, y)+2 h \frac{\partial u(x, y)}{\partial x}+2 h^{2} \frac{\partial^{2} u(x, y)}{\partial x^{2}}+O\left(h^{3}\right)
$$

which leads to

$$
g(x, y)=\frac{1}{2} \frac{\partial u(x, y)}{\partial x}=\frac{-3 u(x, y)-u(x+2 h, y)+4 u(x+h, y)}{4 h}+O\left(h^{2}\right) .
$$

We finally obtain

$u(x, y)=2 g(x, y) h+f(x+h, y) h^{2}+\frac{u(x+h, y+h)+u(x+h, y-h)}{2}+O\left(h^{3}\right)$

which leads to the following Monte Carlo representation. The motion is replaced equiprobably at point $(x+h, y+h)$ or at point $(x+h, y-h)$,

$$
2 g(x, y) h+f(x+h, y) h^{2}
$$

is added to the total score and $h^{2}$ to the total time. This time, the motion is more likely to be in the domain. If not, we divide $h$ iteratively by a factor 2 until it is, as we did for the kinetic approximation.

\section{$5 \quad$ Numerical results}

We shall now test our schemes on two Poisson equations in the square $D=$ $[-1,1]^{2}$ with increasing levels of difficulty. In the first one, we consider a mixed Dirichlet-Neumann where there is no problem of uniqueness for the solution and where the trajectories stop when they hit the "Dirichlet" boundary. This enables a first comparison between the different schemes and validate the new ones introduced in section 4. In the second one, we study the pure Neumann problem for which additional difficulties arise, especially the problems of the uniqueness of the solution and the choice of the time $T_{0}$ to stop the walks. 


\subsection{Mixed Dirichlet-Neumann Poisson equation}

Our first test case is the Poisson equation in the square domain $D=[-1,1]^{2}$ with boundary $\partial D=\partial D_{1} \cup \partial D_{2}$ defined by

$$
\begin{cases}\partial D_{1} & :=\{(x, y),|y|=1,-1 \leq x \leq 1\} \cup\{(x, y), x=-1,-1 \leq y \leq 1\}, \\ \partial D_{2} & :=\{(x, y), x=1,-1 \leq y \leq 1\} .\end{cases}
$$

We have a Neumann boundary condition on $\partial D_{1}$ and a Dirichlet boundary condition on $\partial D_{2}$ :

$$
\begin{cases}-\frac{1}{2} \Delta u(x, y) & =f(x, y):=-\alpha^{2} \exp (\alpha(x+y)) \quad \text { for } \quad(x, y) \in D \\ \frac{1}{2} \frac{\partial u}{\partial n}(x, y) & =g_{1}(x, y):= \pm \frac{\alpha}{2} \exp (\alpha(x+y)) \quad \text { for } \quad(x, y) \in \partial D_{1} \\ u(x, y) & =g_{2}(x, y):=\exp (\alpha(x+y)) \quad \text { for } \quad(x, y) \in \partial D_{2}\end{cases}
$$

where the sign of $g_{1}$ is negative for $y=+1$ and positive otherwise. The stochastic process associated to this equation is a standard Brownian motion $\left(B_{t}, t \geq 0\right)$, reflected on $\partial D_{1}$ and killed on $\partial D_{2}$. The solution $u$ has the stochastic representation

$$
u(x, y)=\mathbb{E}_{(x, y)}\left(\int_{0}^{\tau_{D}} f\left(B_{s}\right) d s+\int_{0}^{\tau_{D}} g_{1}\left(B_{s}\right) d V_{s}+g_{2}\left(B_{\tau_{D}}\right)\right)=\exp (\alpha(x+y))
$$

where $\tau_{D}$ is the first hitting time of $\partial D_{2}$. The parameter $\alpha$ is introduced to obtain solutions with different variations and consequently different variances in the Feynman-Kac representations. We choose the values $\alpha_{1}=\frac{1}{3}, \alpha_{2}=\frac{2}{3}$ and $\alpha_{3}=1$ ranked by increasing degree of difficulty. We compute the solution using $N=50000$ Monte Carlo simulations at some reference points $M_{1}=(0.8,0)$, $M_{2}=(0,0)$ and $M_{3}=(-0.8,0)$ which are located at positions at different distances from $\partial D_{2}$. We also compute the ratio $\frac{\sigma}{\sqrt{N}}$, where $\sigma^{2}$ is the variance of the method which gives an estimation of the Monte Carlo error.

\subsubsection{Euler Scheme approximations}

We denote by $e(\delta, \xi)$ the absolute error on the solution using the reflected Euler scheme with the half space approximation on the Dirichlet side. This error is computed in table 1 with 3 different sets of parameters $\left(\delta_{1}, \xi_{1}\right)=(0.01,0.01)$, $\left(\delta_{2}, \xi_{2}\right)=(0.001,0.000001)$ and $\left(\delta_{3}, \xi_{3}\right)=(0.001,0.001)$.

For the parameters $\left(\delta_{1}, \xi_{1}\right)$, we observe a good accuracy of at least $2.6 \mathrm{E}-2$ on the solution at all the reference points with the various levels of difficulty. We note that the approximate solution is more accurate and the variance smaller at point $M_{1}$ which is very close to the Dirichlet boundary. The approximations at the two other reference points are similar in terms of error and variance. The CPU times on a standard PC for the computation of the solution for the 3 levels of difficulty simultaneously are about 2 seconds for $M_{1}, 7$ seconds for $M_{2}$ and 10 seconds for $M_{3}$. Moreover, variance and bias increase with the level of difficulty. To improve the accuracy of our method, we have first chosen to reduce drastically the regularisation parameter and by a factor 10 the time discretization 


\begin{tabular}{|c|c|c|c|c|c|c|c|}
\hline$(\alpha, M)$ & exact & $e\left(\delta_{1}, \xi_{1}\right)$ & $\frac{\sigma_{1}}{\sqrt{N}}$ & $e\left(\delta_{2}, \xi_{2}\right)$ & $\frac{\sigma_{2}}{\sqrt{N}}$ & $e\left(\delta_{3}, \xi_{3}\right)$ & $\frac{\sigma_{3}}{\sqrt{N}}$ \\
\hline \hline$\left(\alpha_{1}, M_{1}\right)$ & 1.306 & $9.9 \mathrm{E}-4$ & $2.0 \mathrm{E}-3$ & $4.7 \mathrm{E}-3$ & $1.7 \mathrm{E}-2$ & $3.8 \mathrm{E}-4$ & $2.0 \mathrm{E}-3$ \\
\hline$\left(\alpha_{2}, M_{1}\right)$ & 1.705 & $1.8 \mathrm{E}-3$ & $4.6 \mathrm{E}-3$ & $4.6 \mathrm{E}-2$ & $4.2 \mathrm{E}-2$ & $2.1 \mathrm{E}-4$ & $4.7 \mathrm{E}-3$ \\
\hline$\left(\alpha_{3}, M_{1}\right)$ & 2.226 & $1.1 \mathrm{E}-2$ & $8.9 \mathrm{E}-3$ & $1.5 \mathrm{E}-1$ & $8.9 \mathrm{E}-2$ & $2.4 \mathrm{E}-3$ & $9.0 \mathrm{E}-3$ \\
\hline$\left(\alpha_{1}, M_{2}\right)$ & 1 & $1.6 \mathrm{E}-3$ & $3.7 \mathrm{E}-3$ & $6.1 \mathrm{E}-2$ & $3.6 \mathrm{E}-2$ & $3.2 \mathrm{E}-3$ & $3.7 \mathrm{E}-3$ \\
\hline$\left(\alpha_{2}, M_{2}\right)$ & 1 & $9.1 \mathrm{E}-3$ & $8.2 \mathrm{E}-3$ & $1.2 \mathrm{E}-2$ & $8.4 \mathrm{E}-2$ & $9.0 \mathrm{E}-3$ & $8.3 \mathrm{E}-3$ \\
\hline$\left(\alpha_{3}, M_{2}\right)$ & 1 & $2.6 \mathrm{E}-2$ & $1.5 \mathrm{E}-2$ & $6.4 \mathrm{E}-2$ & $1.7 \mathrm{E}-1$ & $1.8 \mathrm{E}-2$ & $1.5 \mathrm{E}-2$ \\
\hline$\left(\alpha_{1}, M_{3}\right)$ & 0.766 & $1.1 \mathrm{E}-2$ & $4.1 \mathrm{E}-3$ & $2.6 \mathrm{E}-2$ & $4.3 \mathrm{E}-2$ & $1.0 \mathrm{E}-3$ & $4.2 \mathrm{E}-3$ \\
\hline$\left(\alpha_{2}, M_{3}\right)$ & 0.587 & $1.6 \mathrm{E}-2$ & $8.9 \mathrm{E}-3$ & $4.7 \mathrm{E}-3$ & $9.6 \mathrm{E}-2$ & $4.7 \mathrm{E}-3$ & $9.0 \mathrm{E}-3$ \\
\hline$\left(\alpha_{3}, M_{3}\right)$ & 0.449 & $1.3 \mathrm{E}-2$ & $1.6 \mathrm{E}-2$ & $7.3 \mathrm{E}-2$ & $1.9 \mathrm{E}-1$ & $1.1 \mathrm{E}-2$ & $1.7 \mathrm{E}-2$ \\
\hline
\end{tabular}

Table 1: Errors in the approximation of the solution of the mixed DirichletNeumann Poisson problem in the domain $D=[-1,1]^{2}$ with an Euler scheme with parameters $\left(\delta_{1}, \xi_{1}\right)=(0.01,0.01),\left(\delta_{2}, \xi_{2}\right)=(0.001,0.000001)$ and $\left(\delta_{3}, \xi_{3}\right)=(0.001,0.001)$.

parameter. The corresponding results for parameters $\left(\delta_{2}, \xi_{2}\right)$ were not satisfactory because the variance increases too much with $\xi_{2}$. This is especially true when $\alpha=\alpha_{3}$ where the accuracy is no more than one digit. The results are a lot better with $\left(\delta_{3}, \xi_{3}\right)$ with roughly the same variance than with $\left(\delta_{1}, \xi_{1}\right)$ but with a smaller bias. For example, the accuracy is 3 times better at point $M_{1}$ for the 3 values of $\alpha$. The CPU times increase by a factor 10 which corresponds to the reduction of $\delta$.

\subsubsection{Walk on spheres approximations}

In table 2 we compare three different methods to compute the absolute errors on the exact solution all relying on the walk on spheres method with absorption parameter $\varepsilon=10^{-6}$ but with different ways to handle the Neumann boundary conditions. The first two errors $F_{3}(h)$ and $F_{2}(h)$ are based on the finite differences method with scores respectively $g(x, y) h+f(x+h, y) h^{2}$ and $g(x, y) h$ at the boundary $\partial D_{1}$. This enables to emphasizes the differences between our new approach with the additional term $f(x+h, y) h^{2}$ and the standard one. The last error $K(h)$ is computed thanks to the kinetic approximation. The simulations are performed with two values $h_{1}=0.2$ and $h_{2}=0.1$ of the parameter $h$. The exit time of the unit circle and the associated uniform position before absorption are pre-computed and stored in files of size $10^{6}$.

The CPU times for the computation of the solution at points $\left(M_{1}, M_{2}, M_{3}\right)$ with discretization step $h_{1}$ are $(4,14,21)$ for the finite differences method, and $(5,20,30)$ for the kinetic approximation (they are twice bigger when using $\left.h_{2}\right)$. In terms of accuracy, we observe that the errors $F_{2}(h)$ are clearly a lot bigger than the ones obtained with the two new methods. The errors $F_{3}\left(h_{2}\right)$ and $K\left(h_{2}\right)$ are similar and are furthermore comparable to $e\left(\delta_{3}, \xi_{3}\right)$ for a CPU time twice smaller. The error $F_{3}\left(h_{1}\right)$ is similar to $e\left(\delta_{1}, \xi_{1}\right)$ for a CPU time twice bigger. We can conclude that the two new schemes are very efficient especially when one 


\begin{tabular}{|c|c|c|c|c|c|c|c|}
\hline$(\alpha, M)$ & $F_{3}\left(h_{1}\right)$ & $F_{3}\left(h_{2}\right)$ & $K\left(h_{1}\right)$ & $K\left(h_{2}\right)$ & $F_{2}\left(h_{1}\right)$ & $F_{2}\left(h_{2}\right)$ & $\frac{\sigma}{\sqrt{N}}$ \\
\hline$\left(\alpha_{1}, M_{1}\right)$ & $9.2 \mathrm{E}-4$ & $2.4 \mathrm{E}-3$ & $4.6 \mathrm{E}-3$ & $1.6 \mathrm{E}-3$ & $1.1 \mathrm{E}-2$ & $4.4 \mathrm{E}-3$ & $2 \mathrm{E}-3$ \\
\hline$\left(\alpha_{2}, M_{1}\right)$ & $6.2 \mathrm{E}-3$ & $5.3 \mathrm{E}-3$ & $2.0 \mathrm{E}-2$ & $1.3 \mathrm{E}-3$ & $3.6 \mathrm{E}-2$ & $1.9 \mathrm{E}-2$ & $4 \mathrm{E}-3$ \\
\hline$\left(\alpha_{3}, M_{1}\right)$ & $1.4 \mathrm{E}-2$ & $7.7 \mathrm{E}-3$ & $5.5 \mathrm{E}-2$ & $3.5 \mathrm{E}-3$ & $9.2 \mathrm{E}-2$ & $4.9 \mathrm{E}-2$ & $8 \mathrm{E}-3$ \\
\hline$\left(\alpha_{1}, M_{2}\right)$ & $1.1 \mathrm{E}-2$ & $2.3 \mathrm{E}-3$ & $3.7 \mathrm{E}-3$ & $2.8 \mathrm{E}-3$ & $5.5 \mathrm{E}-2$ & $2.4 \mathrm{E}-2$ & $4 \mathrm{E}-3$ \\
\hline$\left(\alpha_{2}, M_{2}\right)$ & $1.3 \mathrm{E}-2$ & $1.8 \mathrm{E}-3$ & $3.9 \mathrm{E}-2$ & $3.3 \mathrm{E}-3$ & $1.2 \mathrm{E}-1$ & $8.5 \mathrm{E}-2$ & $8 \mathrm{E}-3$ \\
\hline$\left(\alpha_{3}, M_{2}\right)$ & $1.8 \mathrm{E}-2$ & $9.0 \mathrm{E}-3$ & $1.4 \mathrm{E}-1$ & $2.5 \mathrm{E}-2$ & $4.2 \mathrm{E}-1$ & $2.0 \mathrm{E}-1$ & $1 \mathrm{E}-2$ \\
\hline$\left(\alpha_{1}, M_{3}\right)$ & $1.7 \mathrm{E}-2$ & $1.1 \mathrm{E}-2$ & $2.5 \mathrm{E}-2$ & $1.2 \mathrm{E}-2$ & $6.3 \mathrm{E}-2$ & $4.2 \mathrm{E}-2$ & $4 \mathrm{E}-3$ \\
\hline$\left(\alpha_{2}, M_{3}\right)$ & $1.2 \mathrm{E}-2$ & $1.0 \mathrm{E}-2$ & $4.1 \mathrm{E}-2$ & $1.1 \mathrm{E}-2$ & $2.7 \mathrm{E}-1$ & $1.4 \mathrm{E}-1$ & $8 \mathrm{E}-3$ \\
\hline$\left(\alpha_{3}, M_{3}\right)$ & $1.3 \mathrm{E}-2$ & $6.2 \mathrm{E}-3$ & $1.5 \mathrm{E}-2$ & $3.8 \mathrm{E}-3$ & $5.8 \mathrm{E}-1$ & $3.1 \mathrm{E}-1$ & $2 \mathrm{E}-2$ \\
\hline
\end{tabular}

Table 2: Errors in the approximation of the solution of the mixed DirichletNeumann Poisson problem in the domain $D=[-1,1]^{2}$ with WOS approximations. The errors $F_{3}(h)$ and $F_{2}(h)$ are based on the finite differences method with scores respectively $g(x, y) h+f(x+h, y) h^{2}$ and $g(x, y) h$ at the boundary $\partial D_{2}$. The error $K(h)$ is computed thanks to the kinetic approximation. $h_{1}=0.2$ and $h_{2}=0.1$.

desires an accurate solution. Nevertheless and unlike with Dirichlet boundary conditions, there is not such a big difference in terms of efficiency between Euler schemes and WOS methods.

\subsection{Pure Neumann Poisson Equation}

\subsubsection{Preliminary example}

In this part, we illustrate our theoretical results of section 2 on the solution of a very simple Cauchy problem and of its related variance as a function of $T$. We consider the Poisson equation

$$
\frac{1}{2} \Delta u=-f(x, y)=-2\left(3 x^{2}-1\right)\left(y^{2}-1\right)^{2}-2\left(3 y^{2}-1\right)\left(x^{2}-1\right)^{2}
$$

for $(x, y) \in D=[-1,1]^{2}$ and homogeneous Neumann boundary conditions $\frac{\partial u}{\partial n}=$ 0 on $\partial D$. The stochastic process associated to this equation is a standard reflected Brownian motion and its invariant measure is the uniform law in $D$. The exact solution with mean value zero is

$$
u(x, y)=\left(x^{2}-1\right)^{2}\left(y^{2}-1\right)^{2}-\frac{64}{225} .
$$

In this simple domain, we know that the second leading eigenvalue $\lambda_{1}$ of the operator $-\frac{1}{2} \Delta$ with pure Neumann boundary condition is $\frac{\pi^{2}}{8}$. This means that the convergence of the solution of the Cauchy problem towards the solution of the above equation is a $O\left(\exp \left(-\frac{\pi^{2}}{8} T\right)\right)$. We have also proven that the main part of the variance of our scheme increases linearly as $C_{3} T$, where

$$
C_{3}=\frac{1}{4} \int_{D}|\nabla u|^{2}
$$




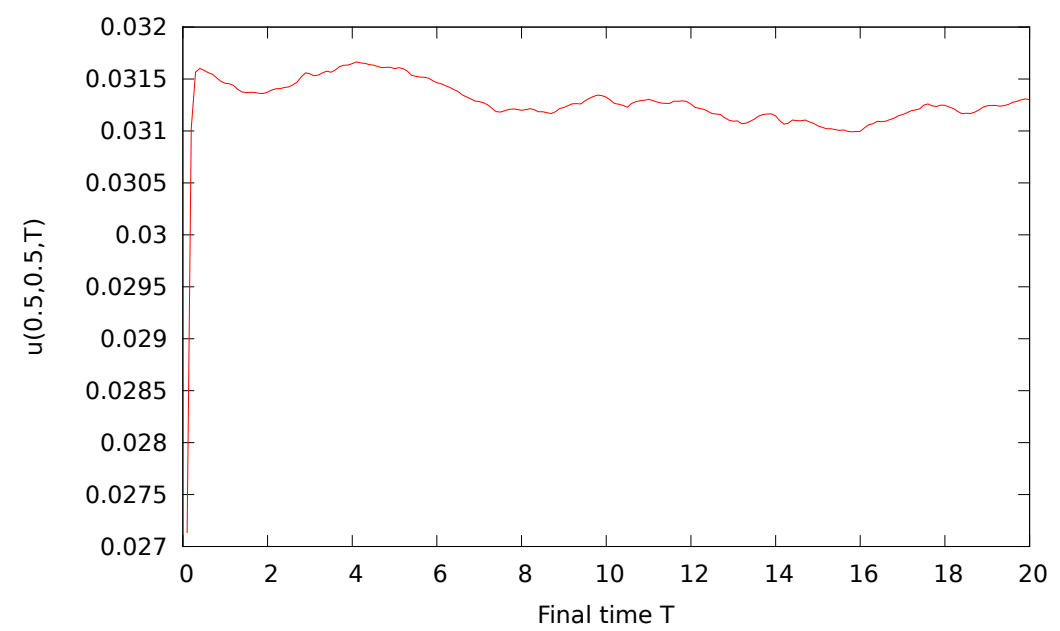

Figure 1: Estimation of the solution of the pure Neumann problem at point $(x, y)=(-0.5,-0.5)$ in function of the final time $T$

Here, we have

$$
|\nabla u|^{2}=\left(4 x\left(x^{2}-1\right)\left(y^{2}-1\right)^{2}\right)^{2}+\left(4 y\left(y^{2}-1\right)\left(x^{2}-1\right)^{2}\right)^{2}
$$

and finally,

$$
C_{3}=\frac{32768}{33075} \simeq 0.99 \text {. }
$$

We compute an approximate solution $u(x, y, T)$ at point $(x, y)=(-0.5,-0.5)$ and its related variance for values of $T \in[0.1,20]$. The numerical method used is the Euler scheme with a small stepsize $\delta=0.001$ and a huge number $N=5 \times 10^{7}$ of simulations. The exact solution with mean value zero is

$$
u(-0.5,-0.5)=\frac{81}{256}-\frac{64}{225} \simeq 0,03196 .
$$

In figure 1, we observe that $u(0.5,0.5, T)$ converges quickly to a constant (modulo some statistical variations) which is close to $u(-0.5,-0.5)$.

Figure 2 concerns the variance: we observe that it increases linearly as a function of $T$. If we compute the slope of the variance using for instance a linear regression on the approximate values at times $T_{i}=8+i$ for $1 \leq i \leq 8$, we obtain about 0.99 . This means that the non linear part which behaves as $C_{2} \sqrt{T}$ should be very small on this particular example.

On this simple example, we have been able to confirm the results obtained in section 2 in the more general setting of a domain in dimension 2. However, we should now consider inhomogeneous Neumann boundary conditions where both bias and variance may increase due to local time approximation. 


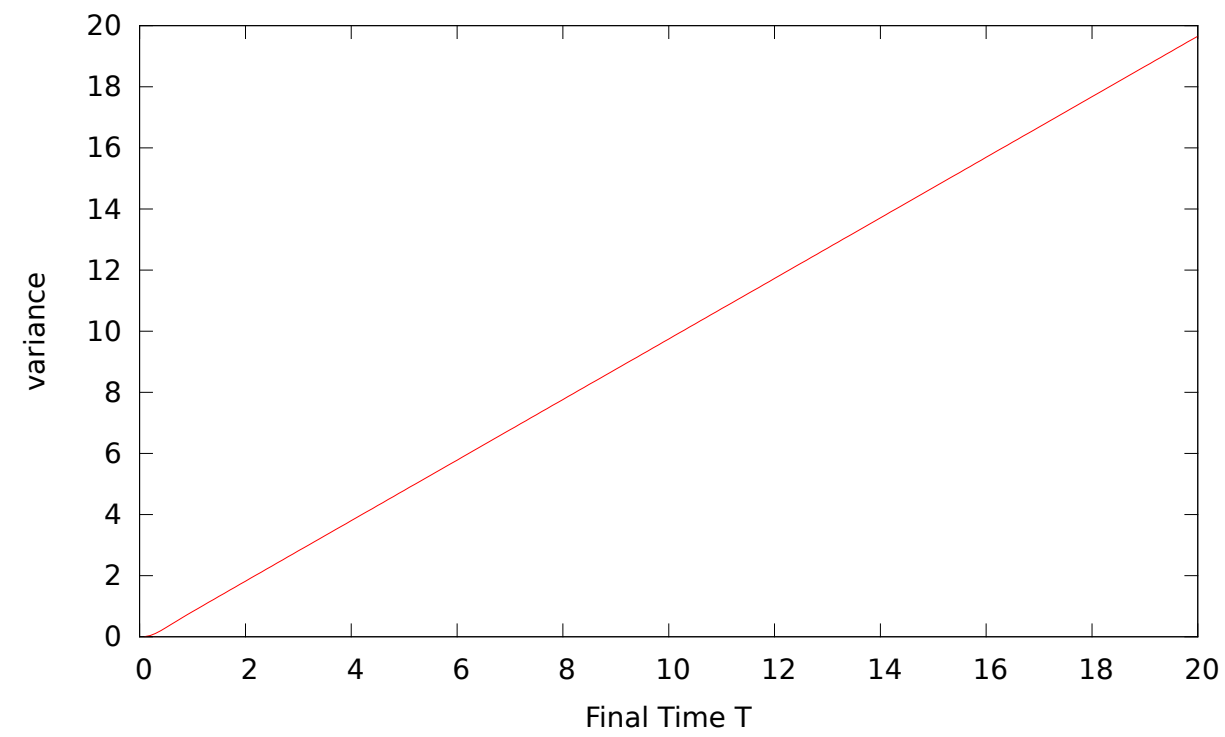

Figure 2: Estimation of the variance of the approximation of the solution of the pure Neumann problem at point $(x, y)=(-0.5,-0.5)$ in function of the final time $T$

\subsubsection{Numerical parameters and error criteria}

Our main test case is the Poisson equation in the square domain $D=[-1,1]^{2}$ defined by

$$
-\frac{1}{2} \Delta u(x, y)=-\alpha^{2} \exp (\alpha(x+y))
$$

in $D$ and with Neumann boundary conditions $\frac{1}{2} \frac{\partial u}{\partial n}= \pm \frac{\alpha}{2} \exp (\alpha(x+y))$ on $\partial D$, where the sign is positive on the bottom and left sides of the boundary and negative on the right and top sides. The solution of this equation with zero mean value with respect to the invariant measure is hence

$$
u(x, y)=\exp (\alpha(x+y))-\frac{1}{4} \int_{-1}^{1} \int_{-1}^{1} \exp (\alpha(x+y)) d x d y
$$

that is

$$
u(x, y)=\exp (\alpha(x+y))-\frac{(\exp (\alpha)-\exp (-\alpha))^{2}}{4 \alpha^{2}} .
$$

This solution is the one that we are likely to obtain numerically if we have a perfect simulation of the reflected Brownian motion and a good choice of the time $T_{0}$ when we stop the trajectories. Even though we have noticed on our test cases on the mixed problem that our numerical schemes are quite efficient, they are obviously not perfect and consequently introduce supplementary errors. In fact, regardless to the usual Monte Carlo and discretization errors, we do 
not compute the solution of the equation with zero mean value with respect to the invariant measure but another one. Nevertheless, we know that our approximation $\bar{u}(x, y)$ should be of the form $u(x, y)+a$ where $a$ is a constant. In order to check the quality of our approximation and estimate this constant, we will compute the minimum of the weighted cost function

$$
J(a)=\frac{1}{\pi^{2}} \int_{-1}^{1} \int_{-1}^{1} \frac{(\bar{u}(x, y)-u(x, y)-a)^{2}}{\sqrt{\left(1-x^{2}\right)\left(1-y^{2}\right)}} d x d y
$$

Using a Gauss quadrature formula on the Tchebychef grid, $J(a)$ can be approximated by

$$
J_{1}(a)=\frac{1}{P^{2}} \sum_{i=1}^{P} \sum_{j=1}^{P}\left(\bar{u}\left(x_{i}, x_{j}\right)-u\left(x_{i}, x_{j}\right)-a\right)^{2}
$$

using the Tchebychef points $x_{i}=\cos \left(\frac{(2 i-1) \pi}{2 P}\right)$. The minimum of $J_{1}$ is achieved for

$$
\bar{a}=\frac{1}{P^{2}} \sum_{i=1}^{P} \sum_{j=1}^{P}\left(\bar{u}\left(x_{i}, x_{j}\right)-u\left(x_{i}, x_{j}\right)\right)
$$

which indicates the bias with respect to the perfectly simulated solution while the value $\rho=\sqrt{J_{1}(\bar{a})}$ quantifies the adequacy to the model. The choice of the weighted cost function is motivated by the use of bidimensional Tchebychef interpolation polynomials in the stochastic spectral methods of the next section. Indeed, they rely on the pointwise approximations at the points of the Tchebychef grid. In practice, we choose $P=3$ which is sufficient to have a good accuracy on the weighted integrals. Once $\bar{a}$ and $\rho$ have been computed, we compute the approximate solution $F(M)=\bar{u}(x, y)$ and the error on the model $B(M)=|\bar{u}(x, y)-u(x, y)-\bar{a}|$ for two sets of points $M$. The first one contains points $M_{4}=(0,0), M_{5}=(-0.2,0.2), M_{6}=(-0.8,0.8)$ and the second one contains points $M_{7}=(0,0.8), M_{8}=(0.2,0.6), M_{9}=(0.4,0.4)$. Morevover all computations are performed using $N=50000$ Monte Carlo simulations.

\subsubsection{Euler scheme approximations}

First of all, we shall choose $T_{0}=10$, which corresponds to a bias equal to $\exp \left(-1.25 \pi^{2}\right) \simeq 5 \times 10^{-6}$ in all the following numerical tests. This value is small enough so that this bias is negligible with respect to the other errors and the variance not too large.

The values of the quantities $\frac{\sigma}{\sqrt{N}}$ depend weakly on the starting point and on the discretization. They are approximatively equal to respectively 0.006 , 0.013 and 0.03 for respectively $\alpha_{1}=\frac{1}{3}, \alpha_{2}=\frac{2}{3}$ and $\alpha_{3}=1$ which is about 1.5 times bigger than in the mixed Dirichlet-Neumann case. When $\left(\delta_{1}, \xi_{1}\right)=$ $(0.01,0.01), \bar{a}$ and $\rho$ are equal respectively to $-0.0059,-0.052,-0.130$ and $0.0039,0.027,0.0498$. When $\left(\delta_{2}, \xi_{2}\right)=(0.001,0.001), \bar{a}$ and $\rho$ are equal respectively to $-0.0015,-0.0042,-0.016$ and $0.0053,0.021,0.042$. 
We observe that the values of $\bar{a}$ and $\rho$ are small which indicates a good adequacy to the model. Moreover, $\rho$ and especially $\bar{a}$ are significantly closer to zero when the discretisation parameters decrease from $\left(\delta_{1}, \xi_{1}\right)$ to $\left(\delta_{2}, \xi_{2}\right)$. This shows that the approximate solution gets closer to $u(x, y)$ as it has been proven in section 2 ,

\begin{tabular}{|c|c|c|c|c|c|c|}
\hline & $F\left(M_{4}\right)$ & $F\left(M_{5}\right)$ & $F\left(M_{6}\right)$ & $B\left(M_{4}\right)$ & $B\left(M_{5}\right)$ & $B\left(M_{6}\right)$ \\
\hline \hline$\left(\alpha_{1},\left(\delta_{1}, \xi_{1}\right)\right)$ & -0.045 & -0.042 & -0.042 & 0.002 & 0.001 & 0.001 \\
\hline$\left(\alpha_{2},\left(\delta_{1}, \xi_{1}\right)\right)$ & -0.204 & -0.172 & -0.210 & 0.005 & 0.038 & 0.001 \\
\hline$\left(\alpha_{3},\left(\delta_{1}, \xi_{1}\right)\right)$ & -0.480 & -0.506 & -0.508 & 0.031 & 0.005 & 0.003 \\
\hline$\left(\alpha_{1},\left(\delta_{2}, \xi_{2}\right)\right)$ & -0.030 & -0.041 & -0.036 & 0.01 & 0.002 & 0.003 \\
\hline$\left(\alpha_{2},\left(\delta_{2}, \xi_{2}\right)\right)$ & -0.155 & -0.182 & -0.146 & 0.006 & 0.02 & 0.016 \\
\hline$\left(\alpha_{3},\left(\delta_{2}, \xi_{2}\right)\right)$ & -0.374 & -0.365 & -0.395 & 0.023 & 0.032 & 0.002 \\
\hline
\end{tabular}

Table 3: Approximation $F$ of the unbiased solution $u_{\bar{a}}$ of the pure Neumann problem with an Euler scheme with steps $\left(\delta_{1}, \xi_{1}\right)=(0.01,0.01)$ and $\left(\delta_{2}, \xi_{2}\right)=$ $(0.001,0.001)$ at points $M_{4}=(0,0), M_{5}=(-0.2,0.2), M_{6}=(-0.8,0.8)$ and the corresponding errors $B(M)=|\bar{u}(M)-u(M)-\bar{a}|$.

On this first set of points, we can see that for the same value of $(\alpha, \delta, \xi)$ the direct estimations $F\left(M_{4}\right), F\left(M_{5}\right)$ and $F\left(M_{6}\right)$ are close to each others. We observe also that the approximation model plays an important role. Indeed, the direct approximations are significantly different from each other for the two sets of parameters but nevertheless the errors $B(M)$ are quite small for both sets. The maximum absolute errors are $0.01,0.038$ and 0.031 for respectively $\alpha_{1}, \alpha_{2}$ and $\alpha_{3}$.

The same conclusions hold for the second set of points. The maximum absolute errors are slightly bigger $0.02,0.054$ and 0.068 for respectively $\alpha_{1}, \alpha_{2}$ and $\alpha_{3}$. These maximum errors are at least 2 or 3 times bigger than in the mixed Dirichlet-Neumann case. Furthermore the CPU times are about twice larger in the pure Neumann Case. We can conclude that the pure Neumann problem is a lot harder to solve but that our algorithm still provides an acceptable accuracy

\begin{tabular}{|c|c|c|c|c|c|c|}
\hline & $F\left(M_{7}\right)$ & $F\left(M_{8}\right)$ & $F\left(M_{9}\right)$ & $B\left(M_{7}\right)$ & $B\left(M_{8}\right)$ & $B\left(M_{9}\right)$ \\
\hline$\left(\alpha_{1},\left(\delta_{1}, \xi_{1}\right)\right)$ & 0.266 & 0.255 & 0.249 & 0.004 & 0.007 & 0.013 \\
\hline$\left(\alpha_{2},\left(\delta_{1}, \xi_{1}\right)\right)$ & 0.550 & 0.524 & 0.526 & 0.054 & 0.028 & 0.031 \\
\hline$\left(\alpha_{3},\left(\delta_{1}, \xi_{1}\right)\right)$ & 0.681 & 0.715 & 0.692 & 0.033 & 0.001 & 0.022 \\
\hline$\left(\alpha_{1},\left(\delta_{2}, \xi_{2}\right)\right)$ & 0.249 & 0.272 & 0.271 & 0.02 & 0.005 & 0.004 \\
\hline$\left(\alpha_{2},\left(\delta_{2}, \xi_{2}\right)\right)$ & 0.549 & 0.537 & 0.518 & 0.006 & 0.006 & 0.025 \\
\hline$\left(\alpha_{3},\left(\delta_{2}, \xi_{2}\right)\right)$ & 0.832 & 0.897 & 0.790 & 0.004 & 0.068 & 0.038 \\
\hline
\end{tabular}

Table 4: Approximation $F$ of the unbiased solution $u_{\bar{a}}$ of the pure Neumann problem with an Euler scheme with steps $\left(\delta_{1}, \xi_{1}\right)=(0.01,0.01)$ and $\left(\delta_{2}, \xi_{2}\right)=$ $(0.001,0.001)$ at points $M_{7}=(0,0.8), M_{8}=(0.2,0.6), M_{9}=(0.4,0.4)$ and the corresponding errors $B(M)=|\bar{u}(M)-u(M)-\bar{a}|$. 


\begin{tabular}{|c|c|c|c|c|c|c|}
\hline & $F\left(M_{4}\right)$ & $F\left(M_{5}\right)$ & $F\left(M_{6}\right)$ & $B\left(M_{4}\right)$ & $B\left(M_{5}\right)$ & $B\left(M_{6}\right)$ \\
\hline$\left(\alpha_{1}, h_{1}\right)$ & -0.055 & -0.059 & -0.062 & 0.002 & 0.001 & 0.004 \\
\hline$\left(\alpha_{2}, h_{1}\right)$ & -0.247 & -0.259 & -0.263 & 0.016 & 0.004 & 0.001 \\
\hline$\left(\alpha_{3}, h_{1}\right)$ & -0.634 & -0.669 & -0.668 & 0.049 & 0.014 & 0.015 \\
\hline$\left(\alpha_{1}, h_{2}\right)$ & -0.043 & -0.050 & -0.048 & 0.006 & 0.001 & 0.001 \\
\hline$\left(\alpha_{2}, h_{2}\right)$ & -0.198 & -0.215 & -0.217 & 0.019 & 0.002 & 0.001 \\
\hline$\left(\alpha_{3}, h_{2}\right)$ & -0.517 & -0.547 & -0.563 & 0.04 & 0.01 & 0.005 \\
\hline
\end{tabular}

Table 5: Approximation $F$ of the unbiased solution $u_{\bar{a}}$ of the pure Neumann problem with the WOS method at points $M_{4}=(0,0), M_{5}=(-0.2,0.2), M_{6}=$ $(-0.8,0.8)$ and the corresponding errors $B(M)=|\bar{u}(M)-u(M)-\bar{a}|$. The parameters are $h_{1}=0.1$ and $h_{2}=0.05$

for pointwise approximations.

\subsubsection{Walk on spheres approximations}

We have noticed in section 5.1 .2 that the two new methods to handle the boundary conditions have the same accuracy. We have chosen to use the order three finite differences in the following. Furthermore to make our simulations, we have precomputed and stored 100 positions of 100000 discretized trajectories as described in section 4.2. The time to open this file is negligible compared to the rest of the simulation times. They are about twice larger than in the mixed Dirichlet-Neumann case. The quantities $\frac{\sigma}{\sqrt{N}}$ are still approximatively equal to respectively $0.006,0.013$ and 0.03 for respectively $\alpha_{1}=\frac{1}{3}, \alpha_{2}=\frac{2}{3}$ and $\alpha_{3}=1$. For $h_{1}=0.1, \bar{a}$ and $\rho$ are equal respectively to $-0.021,-0.106,-0.302$ and $0.018,0.053,0.134$. For $h_{2}=0.05, \bar{a}$ and $\rho$ are equal respectively to -0.012 , $-0.060,-0.176$ and $0.012,0.034,0.085$. We observe that the value of $\rho$ and especially $\bar{a}$ are larger than with the Euler Scheme method.

Once again, the direct estimations $F\left(M_{4}\right), F\left(M_{5}\right)$ and $F\left(M_{6}\right)$ are close to each others for a given value of the parameters $(\alpha, h)$. The maximum absolute errors are $0.006,0.016$ and 0.049 for respectively $\alpha_{1}, \alpha_{2}$ and $\alpha_{3}$. This shows that even if the solution computed is further away from $u(x, y)$, the accuracy is similar than the one obtained with the Euler scheme method.

The maximum absolute errors are bigger for this set of points especially for $\left(\alpha_{3}, h_{1}\right)$. Nevertheless, the maximum absolute errors are $0.011,0.021$ and 0.045 for respectively $\alpha_{1}, \alpha_{2}$ and $\alpha_{3}$. We can conclude that we achieve a good accuracy on this pure Neumann problem but with an increased computational cost compared to the mixed Dirichlet-Neumann problem. 


\begin{tabular}{|c|c|c|c|c|c|c|}
\hline & $F\left(M_{7}\right)$ & $F\left(M_{8}\right)$ & $F\left(M_{9}\right)$ & $B\left(M_{7}\right)$ & $B\left(M_{8}\right)$ & $B\left(M_{9}\right)$ \\
\hline$\left(\alpha_{1}, h_{1}\right)$ & 0.231 & 0.236 & 0.240 & 0.016 & 0.011 & 0.007 \\
\hline$\left(\alpha_{2}, h_{1}\right)$ & 0.399 & 0.412 & 0.424 & 0.043 & 0.03 & 0.018 \\
\hline$\left(\alpha_{3}, h_{1}\right)$ & 0.455 & 0.479 & 0.503 & 0.087 & 0.063 & 0.039 \\
\hline$\left(\alpha_{1}, h_{2}\right)$ & 0.246 & 0.253 & 0.253 & 0.011 & 0.003 & 0.003 \\
\hline$\left(\alpha_{2}, h_{2}\right)$ & 0.466 & 0.475 & 0.474 & 0.021 & 0.012 & 0.013 \\
\hline$\left(\alpha_{3}, h_{2}\right)$ & 0.627 & 0.623 & 0.626 & 0.041 & 0.045 & 0.042 \\
\hline
\end{tabular}

Table 6: Approximation $F$ of the unbiased solution $u_{\bar{a}}$ of the pure Neumann problem with the WOS method at points $M_{7}=(0,0.8), M_{8}=(0.2,0.6)$, $M_{9}=(0.4,0.4)$ and the corresponding errors $B(M)=|\bar{u}(M)-u(M)-\bar{a}|$. The parameters are $h_{1}=0.1$ and $h_{2}=0.05$

\section{Stochastic spectral methods}

\subsection{Spectral formulation}

In this section we describe how to adapt the stochastic spectral formulations introduced in [18] and studied in detail in [19] to the case of pure Neumann boundary conditions. These formulations are similar to usual spectral methods based on polynomial approximations [8] but they are built using relevant information, not necessarily at the collocation points, given by the Feynman-Kac formula. They are an extension of the sequential Monte Carlo algorithms for solving linear partial differential equations developed in [13, 14]. These stochastic spectral formulations are asymptotically perfectly conditioned and quite easy to build for Dirichlet boundary conditions.. The case of mixed boundary conditions is nor described nor studied here because it is a straightforward extension of our previous works. For pure Neumann conditions the situation is quite different because of the non-uniqueness of the solution. We have to build basis functions verifying centering conditions in order to obtain an invertible spectral formulation. These new centering procedures either exact or approximate are described in section 6.2.

When solving the pure Neumann problem using usual deterministic methods like finite elements, one also has to take into account very accurately the compatibility conditions and the non-uniqueness of the solution. Two approaches are usually used. The first one consists in fixing the value of the solution at a specified node in order to avoid the resolution of a singular linear system. The second one leads to a singular system but it is solved using an iterative method like the conjuguate gradient for positive semi-definite linear system. In this second case, it is extremely important that the compatibility condition is verified at the discrete level which is obtained via discrete projectors. All these questions as well as an accurate study of the condition number are treated in [3.

Our stochastic formulation consists in computing a global linear approximation $P_{N} u$ of the solution $u$ using its values at some points $x_{i} \in D$. This global 
approximation writes

$$
P_{N} u(x)=\sum_{i=1}^{N} u\left(x_{i}\right) \Psi_{i}(x)
$$

for some functions $\Psi_{i}(x)$ that are at least twice continuously differentiable and we assume that they verify the centering condition $\int_{D} \Psi_{i}(x) p(x) d x=0$. Note that this last condition implies that $P_{N} u$ belongs to the space of functions that have a zero mean value with respect to $p(x)$ and ensures the uniqueness of the solution. We also assume that for every point $x_{i}$, we can approximate $u\left(x_{i}\right)$ via for instance a numerical approximation of the Feynman-Kac formula by

$$
\widetilde{u}\left(x_{i}\right)=\sum_{j=1}^{q} \alpha_{i, j} g\left(z_{i, j}^{b, \delta, T}\right)+\sum_{j=1}^{p} \beta_{i, j} f\left(z_{i, j}^{s, \delta, T}\right) .
$$

In practice this approximation is also such that

$$
\lim _{p, q, T \rightarrow \infty, \delta \rightarrow 0} \tilde{u}\left(x_{i}\right)=u\left(x_{i}\right)-\int_{D} u(x) p(x) d x
$$

which indicates that the solution that we compute numerically is close to the one with zero mean value with respect to $p(x)$. The coefficients $\alpha_{i, j}$ and $\beta_{i, j}$ are positive weights. The $q$ points $z_{i, 1}^{b, \delta, T}, \cdots, z_{i, q}^{b, \delta, T}$ are located on the boundary $\partial D$, the $p$ points $z_{i, 1}^{s, \delta, T}, \cdots, z_{i, p}^{s, \delta, T}$ in $D, \delta$ stands for the discretization parameter of the simulated reflected diffusion and $T$ is the deterministic time when we decide to stop our random walk. We now let $r_{N}(x)=u(x)-P_{N} u(x)$ and write the partial differential equation solved by $r_{N}(x)$. We have

$$
L r_{N}=L u-L P_{N} u=-f-L P_{N} u
$$

in $D$ with boundary conditions

$$
\frac{\partial r_{N}}{\partial n_{a}}=g-\frac{\partial P_{N} u}{\partial n_{a}}
$$

and hence the approximation

$r_{N}\left(x_{i}\right)=\sum_{j=1}^{q} \alpha_{i, j}\left(g\left(z_{i, j}^{b, \delta, T}\right)-\frac{\partial P_{N} u\left(z_{i, j}^{b, \delta, T}\right)}{\partial n_{a}}\right)+\sum_{j=1}^{p} \beta_{i, j}\left(f\left(z_{i, j}^{s, \delta, T}\right)+L P_{N} u\left(z_{i, j}^{s, \delta, T}\right)\right)$

which leads to the linear system $C u=d$ with

$$
c_{i, i}=\sum_{j=1}^{q} \alpha_{i, j} \frac{\partial \Psi_{i}\left(z_{i, j}^{b, \delta, T}\right)}{\partial n_{a}}-\sum_{j=1}^{p} \beta_{i, j} L \Psi_{i}\left(z_{i, j}^{s, \delta, T}\right)+1-\Psi_{i}\left(x_{i}\right),
$$

for $i \neq k$,

$$
c_{i, k}=\sum_{j=1}^{q} \alpha_{i, j} \frac{\partial \Psi_{k}\left(z_{i, j}^{b, \delta, T}\right)}{\partial n_{a}}-\sum_{j=1}^{p} \beta_{i, j} L \Psi_{k}\left(z_{i, j}^{s, \delta, T}\right)-\Psi_{k}\left(x_{i}\right)
$$


and

$$
d_{i}=\sum_{j=1}^{q} \alpha_{i, j} g\left(z_{i, j}^{b, \delta, T}\right)+\sum_{j=1}^{p} \beta_{i, j} f\left(z_{i, j}^{s, \delta, T}\right) .
$$

As we have done in [18, 19], we can look at the asymptotic system we obtain when $p, q, T \rightarrow \infty$ and when $\delta \rightarrow 0$. The term

$$
\sum_{j=1}^{q} \alpha_{i, j} \frac{\partial \Psi_{k}\left(z_{i, j}^{b, \delta, T}\right)}{\partial n_{a}}-\sum_{j=1}^{p} \beta_{i, j} L \Psi_{k}\left(z_{i, j}^{s, \delta, T}\right)
$$

is our Monte Carlo approximation at point $x_{i}$ of the solution of the equation

$$
L v=L \Psi_{k}
$$

with boundary conditions

$$
\frac{\partial v}{\partial n_{a}}=\frac{\partial \Psi_{k}}{\partial n_{a}}
$$

on $\partial D$ that is $\Psi_{k}\left(x_{i}\right)$ because $\int_{D} \Psi_{k}(x) p(x) d x=0$. As in our previous papers, this shows immediately that the matrix of the asymptotic system converges toward the identity matrix of size $N$. This also means that the condition number of the system is naturally close to one even without additional preconditioning techniques like for instance the ones developed in [28].

\subsection{Centering procedures}

\subsubsection{Exact centering}

We now describe how this method works in practice. The main problem is that in general, usual linear approximations do not verify the centering conditions. We consider for the moment that we start with $N+1$ Lagrange interpolation polynomials $\varphi_{i}$ at $N+1$ points $x_{i} \in D$. Such functions verify $\varphi_{i}\left(x_{k}\right)=\delta_{i, k}$. The usual polynomial interpolation $Q_{N+1} u$ of degree $N$ of a function $u$ writes $Q_{N+1} u(x)=\sum_{i=1}^{N+1} u\left(x_{i}\right) \varphi_{i}(x)$. Considering the constant function $u=1$, we obviously have $\sum_{i=1}^{N+1} \varphi_{i}(x)=1$ and hence

$$
\sum_{i=1}^{N+1} \int_{D} \varphi_{i}(x) p(x) d x=1
$$

which proves that this usual interpolation cannot verify the centering conditions. Nevertheless, we can choose an index $i_{0}$ such that

$$
\left|\int_{D} \varphi_{i_{0}}(x) p(x) d x\right|=\max _{1 \leq i \leq N+1}\left|\int_{D} \varphi_{i}(x) p(x) d x\right| \neq 0
$$

for a sake of stability in the following approximation. The centering condition for $Q_{N+1} u$ writes

$$
\sum_{i=1}^{N+1}\left(\int_{D} \varphi_{i}(x) p(x) d x\right) u\left(x_{i}\right)=0
$$


which leads to

$$
Q_{N+1} u(x)=\sum_{i \neq i_{0}} u\left(x_{i}\right)\left(\varphi_{i}(x)-\frac{\int_{D} \varphi_{i}(x) p(x) d x}{\int_{D} \varphi_{i_{0}}(x) p(x) d x} \varphi_{i_{0}}(x)\right) .
$$

Letting now

$$
\Psi_{i}(x)=\varphi_{i}(x)-\frac{\int_{D} \varphi_{i}(x) p(x) d x}{\int_{D} \varphi_{i_{0}}(x) p(x) d x} \varphi_{i_{0}}(x),
$$

the new basis functions verify the centering conditions $\int_{D} \Psi_{i}(x) p(x) d x=0$ and still $\Psi_{i}\left(x_{k}\right)=\delta_{i, k}$. This centering procedure can be easily extended to general linear approximations not necessarily of interpolation type. It is essentially the same approach than the projection method described in [3] for the finite elements method.

\subsubsection{Approximate centering}

This solves our problem whenever the integrals $\int_{D} \varphi_{i}(x) p(x) d x$ can be computed exactly. This may happen when the domain is simple and when the density $p(x)$ is known. This include for example the case of the Poisson equation in a hypercube using an interpolation on a Tchebychef grid. In many other cases, these integrals need to be computed numerically. The density $p(x)$ can be approximated by the law of the position $Y$ of $M$ particles moving according to the reflected diffusion starting at any given point in $D$ at a time $T_{1}$ large enough. We obtain

$$
\int_{D} \varphi_{i}(x) p(x) d x \simeq \frac{1}{M} \sum_{j=1}^{M} \varphi_{i}\left(Y_{j}\right)
$$

and new basis functions which are defined by

$$
\Psi_{i}(x)=\varphi_{i}(x)-\frac{\frac{1}{M} \sum_{j=1}^{M} \varphi_{i}\left(Y_{j}\right)}{\frac{1}{M} \sum_{j=1}^{M} \varphi_{i_{0}}\left(Y_{j}\right)} \varphi_{i_{0}}(x) .
$$

The coefficients of the asymptotic spectral matrix are

$$
\left\{\begin{array}{l}
c_{i, i}=1-\int_{D} \Psi_{i}(x) p(x) d x \\
c_{i, j}=-\int_{D} \Psi_{i}(x) p(x) d x \text { for } i \neq j .
\end{array}\right.
$$

An easy computation shows that its eigenvalues are all but one equal to one. The remaining eigenvalue is

$$
\lambda_{N}=1-\sum_{i=1}^{N} \int_{D} \Psi_{i}(x) p(x) d x
$$

and we have

$$
1-\lambda_{N}=\sum_{i=1}^{N}\left(\int_{D} \varphi_{i}(x) p(x) d x-\frac{\frac{1}{M} \sum_{j=1}^{M} \varphi_{i}\left(Y_{j}\right)}{\frac{1}{M} \sum_{j=1}^{M} \varphi_{i_{0}}\left(Y_{j}\right)} \int_{D} \varphi_{i_{0}}(x) p(x) d x\right)
$$


and the inequality

$$
\begin{aligned}
\left|1-\lambda_{N}\right|\left|\frac{1}{M} \sum_{j=1}^{M} \varphi_{i_{0}}\left(Y_{j}\right)\right| \leq & \left|\int_{D} \sum_{i=1}^{N} \varphi_{i}(x) p(x) d x-\frac{1}{M} \sum_{j=1}^{M} \sum_{i=1}^{N} \varphi_{i}\left(Y_{j}\right)\right|\left|\int_{D} \varphi_{i_{0}}(x) p(x) d x\right| \\
& \left.+\mid \int_{D} \varphi_{i_{0}}(x) p(x) d x\right)-\frac{1}{M} \sum_{j=1}^{M} \varphi_{i_{0}}\left(Y_{j}\right)|| \int_{D} \sum_{i=1}^{N} \varphi_{i}(x) p(x) d x \mid
\end{aligned}
$$

which proves that $\lambda_{N}$ converges to 1 when $M \rightarrow \infty$ and that the condition number is once again asymptotically one. Note that we compute with this formulation an approximation

$$
P_{N} u(x)=\sum_{i=1}^{N} u\left(x_{i}\right) \Psi_{i}(x)
$$

of the solution with discrete integral equal to zero with respect to the particle approximation of $p(x)$.

\subsection{Application to the Poisson equation}

In this section, we describe the application of the stochastic spectral formulation on our main example of the Poisson equation with pure Neumann boundary conditions studied in section 5 . The basis functions will rely on Tchebychef interpolation polynomials in dimension 2 . The big advantage of this test case is that the centering procedure can be done exactly as we know that the invariant probability is the uniform law and because the integration domain is a square. This also enables us to compare the exact centering procedure and the approximate one where the numerical integration is done by means of a particle approximation of the invariant measure.

\subsubsection{Basis functions}

Our spectral approximation is based on the standard tensorized interpolation of the solution on the Tchebychef grid which writes

$$
\sum_{n=0}^{N} \sum_{m=0}^{N} u_{n, m} l_{n}(x) l_{m}(y)
$$

where $l_{n}$ is the Lagrange polynomial associated to $z_{n}$ and $u_{n, m}$ is the approximate value of the solution at the point $\left(z_{n}, z_{m}\right)$ where $z_{n}=\cos \left(\frac{2 n+1}{2 N+2} \pi\right)$, $0 \leq n \leq N$. For the sake of simplicity, we choose $N$ even so that point $(0,0)$ belongs to the Tchebychef grid. Indeed, in this case the maximum of the integrals

$$
\int_{-1}^{1} \int_{-1}^{1} l_{n}(x) l_{m}(y) d x d y
$$




\begin{tabular}{|c|c|c|c|c|}
\hline & $e r r_{1}\left(N_{1}\right)$ & $\kappa\left(N_{1}\right)$ & $e r r_{1}\left(N_{2}\right)$ & $\kappa\left(N_{2}\right)$ \\
\hline$\left(\delta_{1}, M_{1}\right)$ & $6.1 \mathrm{E}-3$ & 11.4 & $3.6 \mathrm{E}-5$ & 856 \\
\hline$\left(\delta_{1}, M_{2}\right)$ & $3.4 \mathrm{E}-3$ & 2.9 & $1.2 \mathrm{E}-5$ & 121 \\
\hline$\left(\delta_{2}, M_{1}\right)$ & $4.2 \mathrm{E}-3$ & 5.1 & $2.3 \mathrm{E}-5$ & 114 \\
\hline$\left(\delta_{2}, M_{2}\right)$ & $1.1 \mathrm{E}-3$ & 2.3 & $3.1 \mathrm{E}-6$ & 17 \\
\hline
\end{tabular}

Table 7: Global error $\operatorname{err}_{1}(N)=\max _{0 \leq i, j \leq N}\left|u\left(z_{i}, z_{j}\right)-u_{i, j}\right|$ of the approximation of the solution of the pure Neumann problem with Euler scheme with time steps $\delta_{1}=0.01, \delta_{2}=0.001$, and a regularization parameter $\xi=0.001$ for for $N_{1}=2$ and $N_{2}=4$ and $\alpha=\frac{1}{3}$. The value of condition number of the spectral matrix is $\kappa(N)$.

is always attained for the Lagrange polynomials $l_{\frac{N}{2}}(x)$ and $l_{\frac{N}{2}}(y)$ corresponding to this point. The function $l_{\frac{N}{2}}(x) l_{\frac{N}{2}}(y)$ is removed from the basis functions and the $(N+1)^{2}-1$ centered basis functions $\Psi_{n, m}(x, y)$ now write

$$
\Psi_{n, m}(x, y)=l_{n}(x) l_{m}(y)-\frac{\int_{-1}^{1} \int_{-1}^{1} l_{n}(x) l_{m}(y) d x d y}{\int_{-1}^{1} \int_{-1}^{1} l_{\frac{N}{2}}(x) l_{\frac{N}{2}}(y) d x d y} l_{\frac{N}{2}}(x) l_{\frac{N}{2}}(y)
$$

for $(n, m) \neq\left(\frac{N}{2}, \frac{N}{2}\right)$. In the case of the approximate centering, the integrals above are replaced by their particle approximations.

\subsubsection{Numerical results: exact centering}

In table 77 we present our results based on the Euler scheme approximation with two time discretization parameters $\delta_{1}=0.01, \delta_{2}=0.001$, a regularization parameter $\xi=0.001$ and with two different numbers of simulations $M_{1}=1000$ and $M_{2}=5000$. The trajectories are stopped at final time $T_{0}=10$. For these four sets of parameters, we compute the maximum absolute error over the grid points

$$
\operatorname{err}_{1}(N)=\max _{0 \leq i, j \leq N}\left|u\left(z_{i}, z_{j}\right)-u_{i, j}\right|
$$

and the condition number $\kappa(N)$ of the spectral matrix for $N_{1}=2$ and $N_{2}=4$ and $\alpha=\frac{1}{3}$.

We can observe that the condition number $\kappa$ is decreasing as $M$ increases and $\delta$ decreases. The system is very well conditioned especially for the parameters $\left(\delta_{2}, M_{2}\right)$. As for usual standard spectral methods, the error is small and decreases with $\kappa$ and when $N$ increases.

\subsubsection{Numerical results: approximate centering}

The spectral method now requires the approximation of the invariant measure which should be done with $Q$ simulations and a time step $\delta$. The invariant measure is the uniform law in the square $[-1,1]^{2}$. To study its impact on the spectral matrix, we have chosen in table 8 to make its approximation using 


\begin{tabular}{|c|c|c|c|c|c|c|}
\hline & $\operatorname{err}_{1}\left(N_{1}\right)$ & $\operatorname{err}_{2}\left(N_{1}\right)$ & $\kappa\left(N_{1}\right)$ & $\operatorname{err}_{1}\left(N_{2}\right)$ & $\operatorname{err}_{2}\left(N_{2}\right)$ & $\kappa\left(N_{2}\right)$ \\
\hline$\left(\delta_{1}, M_{1}, Q_{1}\right)$ & $1.9 \mathrm{E}-2$ & $2.4 \mathrm{E}-3$ & 10.2 & $4.60 \mathrm{E}-2$ & $2.7 \mathrm{E}-5$ & 550 \\
\hline$\left(\delta_{1}, M_{1}, Q_{2}\right)$ & $5.8 \mathrm{E}-3$ & $5.1 \mathrm{E}-3$ & 26.3 & $1.5 \mathrm{E}-3$ & $2.3 \mathrm{E}-4$ & 1187 \\
\hline$\left(\delta_{1}, M_{2}, Q_{1}\right)$ & $1.7 \mathrm{E}-2$ & $1.6 \mathrm{E}-3$ & 5.2 & $1.4 \mathrm{E}-2$ & $6.3 \mathrm{E}-5$ & 1130 \\
\hline$\left(\delta_{1}, M_{2}, Q_{2}\right)$ & $5.1 \mathrm{E}-3$ & $6.1 \mathrm{E}-4$ & 2.9 & $3.1 \mathrm{E}-3$ & $1.0 \mathrm{E}-5$ & 644 \\
\hline$\left(\delta_{2}, M_{1}, Q_{1}\right)$ & $1.6 \mathrm{E}-2$ & $3.6 \mathrm{E}-3$ & 4.1 & $1.5 \mathrm{E}-2$ & $5.4 \mathrm{E}-5$ & 309 \\
\hline$\left(\delta_{2}, M_{1}, Q_{2}\right)$ & $3.9 \mathrm{E}-3$ & $1.4 \mathrm{E}-3$ & 3.0 & $7.4 \mathrm{E}-4$ & $1.4 \mathrm{E}-5$ & 252 \\
\hline$\left(\delta_{2}, M_{2}, Q_{1}\right)$ & $6.3 \mathrm{E}-2$ & $9.4 \mathrm{E}-4$ & 2.8 & $3.0 \mathrm{E}-2$ & $3.1 \mathrm{E}-6$ & 22 \\
\hline$\left(\delta_{2}, M_{2}, Q_{2}\right)$ & $1.5 \mathrm{E}-3$ & $1.2 \mathrm{E}-3$ & 1.6 & $3.3 \mathrm{E}-3$ & $4.2 \mathrm{E}-6$ & 16 \\
\hline
\end{tabular}

Table 8: Global approximation of the solution of the pure Neumann problem with an Euler scheme. The grid parameters are respectively $N_{1}=2$ and $N_{2}=4$. The time steps are $\delta_{1}=0.01, \delta_{2}=0.001$. The invariant measure is approximated with samples of sizes $Q_{1}=100$ and $Q_{2}=10000$.

simply $Q$ samples $\left(X_{l}, Y_{l}\right)$ of this uniform law. We use two samples of different sizes $Q_{1}=100$ and $Q_{2}=10000$ and introduce a new error criterion

$$
\operatorname{err}_{2}(N)=\max _{0 \leq i, j \leq N}\left|u\left(z_{i}, z_{j}\right)-u_{i, j}+\frac{1}{4 Q} \sum_{l=1}^{Q} u\left(X_{l}, Y_{l}\right)\right|
$$

as we have proven that we approximate the solution with discrete integral equal to zero with respect to the particle approximation of $p(x)$. We nevertheless keep also the previous error criterion to study the impact of the particle approximation on the bias.

The key observation is that we check that the solution effectively computed is the one with discrete integral equal to zero with respect to the particle approximation of $p(x)$. Both accuracy and condition number have the same behaviour with respect to the parameters $\delta$ and $M$ than with the exact centering. The condition number and $\operatorname{err}_{1}$ decrease when the particle approximation is done with more points.

\subsection{Application to a convection-diffusion problem}

In this section, we study a more general convection diffusion equation with an additional drift coefficient depending assymmetrically on the spatial position. We keep nevertheless a square domain in order not to mix the approximation problems in a general bounded domain and the other difficulties met by our method. We perform our algorithm on the operator

$$
L=\frac{1}{2} \Delta+x \beta_{x} \frac{\partial}{\partial x}+y \beta_{y} \frac{\partial}{\partial y}
$$

with a source term $f(x, y)=\alpha\left(\alpha+x \beta_{x}+y \beta_{y}\right) \exp (\alpha(x+y))$ and a boundary term $g(x, y)= \pm \frac{\alpha}{2} \exp (\alpha(x+y))$ on $\partial D$ such that the solution up to an additive constant is still $\exp (\alpha(x+y))$. For this model, we do not know the exact invariant 


\begin{tabular}{|c|c|c|c|c|}
\hline & $e r r_{2}\left(N_{1}\right)$ & $\kappa\left(N_{1}\right)$ & $\operatorname{err}_{2}\left(N_{2}\right)$ & $\kappa\left(N_{2}\right)$ \\
\hline$\left(\delta_{1}, M_{1}, Q_{1}\right)$ & $4.7 \mathrm{E}-3$ & 16.1 & $2 . \mathrm{E}-5$ & 2648 \\
\hline$\left(\delta_{1}, M_{1}, Q_{2}\right)$ & $2.6 \mathrm{E}-3$ & 26.4 & $1.1 \mathrm{E}-5$ & 649 \\
\hline$\left(\delta_{1}, M_{2}, Q_{1}\right)$ & $1.1 \mathrm{E}-2$ & 74.2 & $1.0 \mathrm{E}-5$ & 1254 \\
\hline$\left(\delta_{1}, M_{2}, Q_{2}\right)$ & $3.1 \mathrm{E}-3$ & 12.0 & $4.3 \mathrm{E}-4$ & 22437 \\
\hline$\left(\delta_{2}, M_{1}, Q_{2}\right)$ & $2.7 \mathrm{E}-3$ & 5.9 & $2.1 \mathrm{E}-5$ & 1200 \\
\hline$\left(\delta_{2}, M_{2}, Q_{2}\right)$ & $8.1 \mathrm{E}-4$ & 4.0 & $2.0 \mathrm{E}-6$ & 39.8 \\
\hline
\end{tabular}

Table 9: Global approximation of the solution of the pure Neumann problem with an Euler scheme. The grid parameters are respectively $N_{1}=2$ and $N_{2}=4$. The time steps are $\delta_{1}=0.01, \delta_{2}=0.001$. The invariant measure is approximated with samples of sizes $Q_{2}=10000$ and $Q_{1}=100$ only for $\delta_{1}$. The final time $Q_{1} \delta_{2}=0.1$ is not large enough to reasonnably approximate the invariant measure.

measure of the reflected diffusion process associated to the operator $L$. To compute its particle approximation, we run a single path starting at the center of the domain with a time step $\delta$ and ending at time $T=Q \delta$. The $Q$ points of this path give an approximation of the invariant measure. We present in table 9 our results for the parameters $\beta_{x}=0.2, \beta_{y}=0.1$ and $\alpha=0.3$. We observe that the accuracy of the algorithm is still very high, the condition number of the system still very low even if we approximate the invariant measure by running a path. This confirms that our approach is quite general.

\section{Conclusion}

To compute Monte Carlo approximations of the solution of the Neumann problem for elliptic equations, we had to overcome several difficulties. First, we have characterized the solution of the Feynman-Kac representation introduced in 2 . as the one with zero mean value with respect to the invariant measure of its associated stochastic process. Then, we have proven that the variance increases mainly linearly as a function of the time $T_{0}$ to stop the trajectories.

We have introduced some new schemes to deal with the inhomogeneous Neumann boundary conditions. They were tested first on a pointwise approximations of mixed Dirichlet-Neumann problem where they show a good efficiency. The pointwise resolution of the pure Neumann problem was a lot harder. Indeed, we had to choose $T_{0}$ not too large because of the increase of the variance but also not too small because of the bias. We had also to understand that the solution computed numerically depends on the parameters of the numerical schemes and is not equal to the one with zero mean value with respect to the invariant measure. Taken all these difficulties into account, we have been able nevertheless to reach a reasonable accuracy on the approximate solutions.

Concerning the global spectral approximation, we had to pay attention to the zero mean value property of the solution to chose our approximation basis. This has been achieved using exact or approximate centering procedures very 
similar to the usual ones used in finite element methods. In both cases, the condition number of the spectral matrix was proven to be asymptotically one. The numerical experiments show that the stochastic spectral method is both very accurate and well-conditioned.

The pointwise approximations of the pure Neumann problem are not completely satisfactory because the solutions obtained depend on the parameters of the numerical scheme. The choice of the time $T_{0}$ to stop the trajectories is not straightforward for a general diffusion in a complex domain. For the WOS method, it requires furthermore to keep in memory discretisation of trajectories which is both costly and adds an error not so easy to quantify. It could be interesting to add a penalization term either in the source term or via Robin boundary conditions to at least get rid of some of these drawbacks.

\section{Acknowledgments}

We would like to thank the Anonymous Referee for his/her constructive comments on our work and for drawing our attention to some references we were not aware of.

\section{References}

[1] R. F. BASS, P. HSU, Some potential theory for reflecting Brownian motion in Hölder and Lipschitz domains, The Annals of Probability 19 (2), 486-508, 1991.

[2] A. BENCHÉRIF-MADANI, E. PARDOUX, A probabilistic formula for a Poisson equation with Neumann boundary condition, Stoch. Anal. Appl. 27, 739-746, 2009.

[3] P. BOCHEV, R. B. LEHOUCQ, On the finite element solution of the pure Neumann problem, SIAM Review 47, 50-66, 2005.

[4] M. BOSSY, N. CHAMPAGNAT, S. MAIRE, D. TALAY, Probabilistic interpretation and random walk on spheres algorithms for the PoissonBoltzmann equation in molecular dynamics, Mathematical Modelling and Numerical Analysis 44 (5), 997-1048, 2010.

[5] M. BOSSY, E. GOBET, and D. TALAY, A symmetrized Euler scheme for an efficient approximation of reflected diffusions, J. Appl. Probab. 41 (3), 877-889, 2004.

[6] G. A. BROSAMLER, A probabilistic solution of the Neumann problem, Math. Scand. 38, 137-147, 1976.

[7] K. BURDZY, Neumann eigenfunctions and Brownian couplings, Proc. Potential theory in Matsue, Adv. Stud. Pure Math., Math. Soc. Japan, Tokyo 44, 11-23, 2006. 
[8] C. CANUTO, M. Y. HUSSAINI, A. QUARTERONI, T. A. ZANG, Spectral methods in fluid dynamics. Springer-Verlag, 1988.

[9] M. DEACONU, A. LEJAY, A random walk on rectangles algorithms, Methodol. Comput. Appl. Probab. 8 (1), 135-151, 2006.

[10] G. GIRAUD, Problèmes mixtes et Problèmes sur des variétés closes, relativement aux équations linéaires du type elliptique, Ann. Soc. Polon. Math. 12, 35-53, 1933.

[11] E. GOBET, Weak approximations of killed diffusions using Euler schemes. Stochastic processes and their applications, 87, 167-197, 2000.

[12] E. GOBET, Euler schemes and half-space approximations for the simulation of diffusion in a domain, ESAIM Probability and Statistics, 5, 261-297, 2001.

[13] E. GOBET, S. MAIRE, A spectral Monte Carlo method for the Poisson equation. Monte Carlo Methods and applications 10 (3-4), 275-285, 2004.

[14] E. GOBET, S. MAIRE, Sequential control variates for functionals of Markov processes, SIAM Journal on Numerical Analysis 43 (3), 1256-1275, 2005 .

[15] C. HWANG, M. MASCAGNI, J.A. GIVENS. A Feynman-Kac pathintegral implementation for Poisson's equation using an h-conditioned Green function. Mathematics and Computers in Simulation 62, 347-355, 2003.

[16] G. J. JIANG and J. L. KNIGHT. A nonparametric approach to the estimation of diusion processes, with an application to a short-term interest rate model. Econometric Theory, 13 (5), 615-645, 1997.

[17] A. LEJAY, S. MAIRE, Simulating diffusions with piecewise constant coefficients using a kinetic approximation, Computer Methods in Applied Mechanics and Engineering 199 (29-32), 2014-2023, 2010.

[18] S. MAIRE, E. TANRÉ, Some new simulation schemes for the evaluation of Feynman-Kac representations, Monte Carlo methods and applications 14 (1), 29-51, 2008.

[19] S. MAIRE, E. TANRÉ, Stochastic spectral formulations for elliptic problems, Monte Carlo and Quasi-Monte Carlo 2008, P. L'Ecuyer, A. Owen (Eds), Springer, 513-528, 2009.

[20] M. MASCAGNI, N. SIMONOV, Monte Carlo methods for calculating some physical properties of large molecules, SIAM journal on scientific computing, 26 (1), 339-357, 2004. 
[21] G.A MIKHAILOV, R.N MAKAROV, Solution of boundary value problems of the second and third kind by the Monte Carlo method, Sib. Mat. Zh. 38, 603-614, 1997.

[22] G. N. MILSTEIN and M.V. TRETYAKOV, Stochastic Numerics for Mathematical Physics, Springer, 2004.

[23] V. REUTENAUER, E.TANRÉ, Exact simulation of prices and greeks: application to CIR, Preprint HAL-Inria, 2008.

[24] K. SABELFELD, Monte Carlo methods in boundary value problems. Springer, Berlin, 1991.

[25] K. K. SABELFELD and N. A. SIMONOV, Random walks on boundary for solving PDEs, VSP, 1994.

[26] N. A. SIMONOV, Walk-on-Spheres algorithm for solving boundary-value problems with continuity flux conditions, Monte Carlo and Quasi-Monte Carlo 2006, Springer-Verlag, Heidelberg, 2007, pp. 633-644.

[27] D. TALAY, L. TUBARO, Expansion of the global error for numerical schemes solving stochastic differential equations, Stochastic analysis and applications, 8 (4), 483-509, 1990.

[28] J. M. TANG, C. VUIK, On deflation and singular symmetric positive semidefinite matrices, Journal of computational and applied mathematics 206, 603-614, 2007. 